Supporting Information for

\title{
Interfacial Synthesis of Layer-Oriented 2D Conjugated Metal-Organic Framework Films toward Directional Charge Transport
}

Zhiyong Wang ${ }^{1}$, Lisa S. Walter ${ }^{2,7}$, Mao Wang ${ }^{3}$, Petko St. Petkov ${ }^{4}$, Baokun Liang, Haoyuan Qi ${ }^{1,5}$, Nguyen Ngan Nguyen ${ }^{1}$, Mike Hambsch ${ }^{11}$, Haixia Zhong ${ }^{1}$, Mingchao Wang ${ }^{1}$, SangWook Park ${ }^{1}$, Lukas Renn ${ }^{2,7}$, Kenji Watanabe ${ }^{6}$, Takashi Taniguchi ${ }^{6}$, Stefan C. B. Mannsfeld ${ }^{11}$, Thomas Heine ${ }^{1,9,10}$, Ute Kaiser ${ }^{5}$, Shengqiang Zhou ${ }^{3}$, Ralf Thomas Weitz $^{2,7, *}$, Xinliang Feng ${ }^{1,8, *}$ and Renhao Dong ${ }^{1 *}$

${ }^{1}$ Center for Advancing Electronics Dresden (cfaed) and Faculty of Chemistry and Food Chemistry, Technische Universität Dresden, 01062 Dresden, Germany

${ }^{2}$ I. Physical Institute, Faculty of Physics, Georg-August-University Göttingen, 37077 Göttingen, Germany

${ }^{3}$ Helmholtz-Zentrum Dresden-Rossendorf, Institute of Ion Beam Physics and Materials Research, 01328 Dresden, Germany

${ }^{4}$ Faculty of Chemistry and Pharmacy, University of Sofia, 1164 Sofia, Bulgaria

${ }^{5}$ Central Facility for Electron Microscopy, Electron Microscopy of Materials Science Central, Facility for Electron Microscopy, Ulm University, 89081 Ulm, Germany ${ }^{6}$ National Institute for Materials Science, 305-0047 Tsukua, Japan

${ }^{7}$ Physics of Nanosystems, Department of Physics, Ludwig-Maximilians-University München, 80799 Munich, Germany

${ }^{8}$ Max Planck Institute for Microstructure Physics, Weinberg 2, Halle (Saale), D-06120 Germany

${ }^{9}$ Helmholtz-Zentrum Dresden-Rossendorf, Institute of Resource Ecology, Leipzig Research Branch, 04316 Leipzig, Germany

${ }^{10}$ Department of Chemistry, Yonsei University, 50 Yonsei-ro, Seodaemun-gu, Seoul, 03722 Korea

${ }^{11}$ Center for Advancing Electronics Dresden (cfaed) and Faculty of Electrical and Computer Engineering, Technische Universität Dresden, 01062 Dresden, Germany

\section{Corresponding Author}

*Ralf Thomas Weitz (thomas.weitz@uni-goettingen.de)

*Xinliang Feng (xinliang.feng@tu-dresden.de)

*Renhao Dong (renhao.dong@tu-dresden.de) 


\section{Table of Contents}

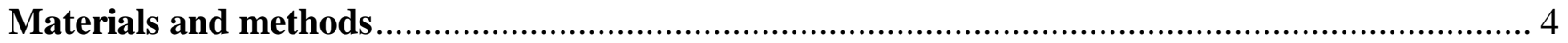

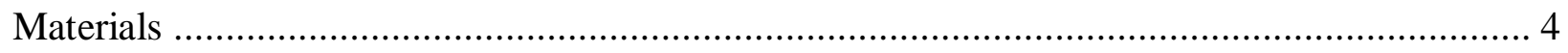

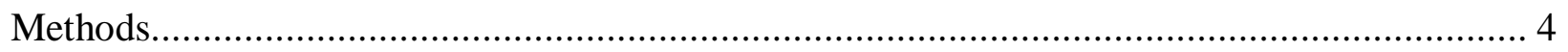

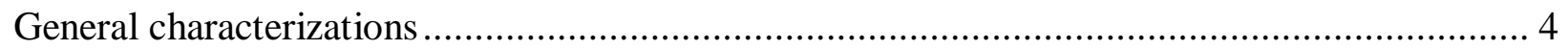

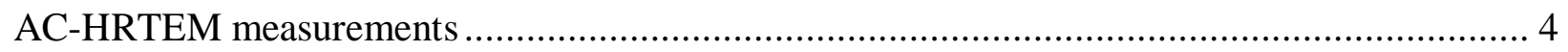

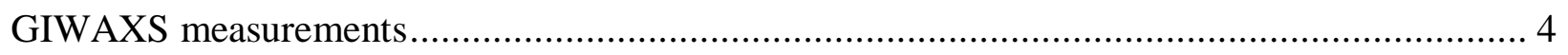

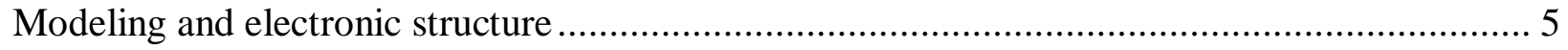

Variable-temperature conductivity and Hall effect measurements in macroscopic devices for

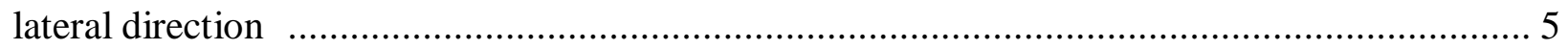

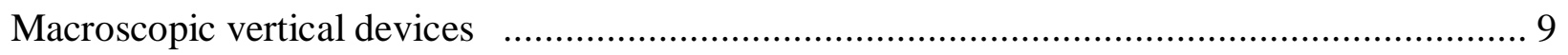

Local characterization of directional charge transport .................................................. 9

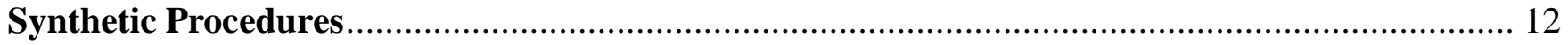

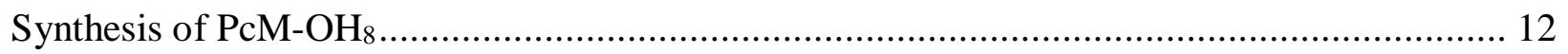

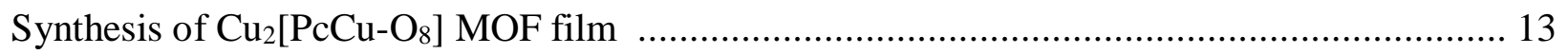

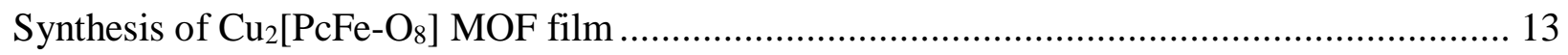

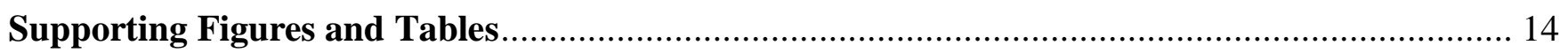

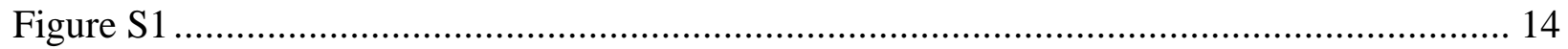

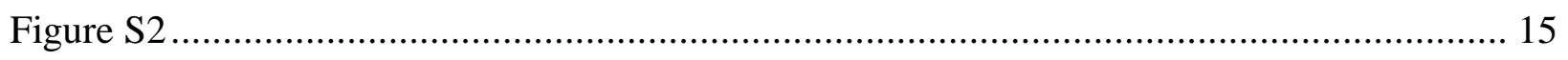

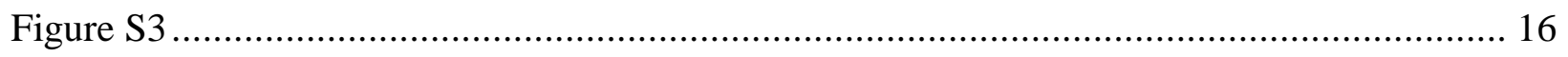

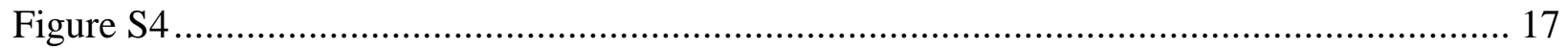

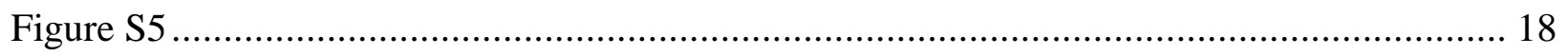

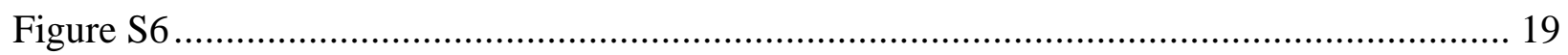

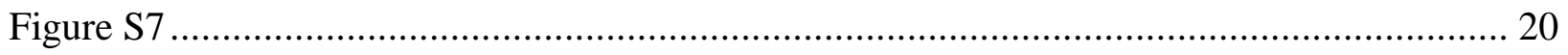

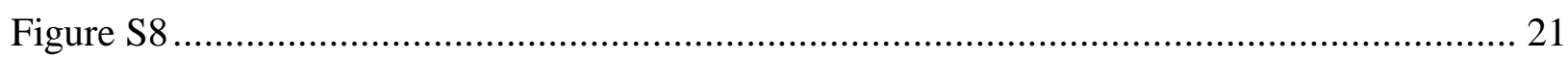

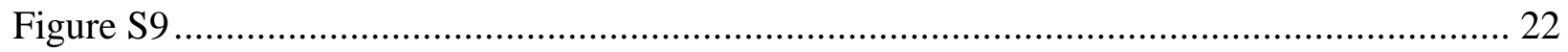

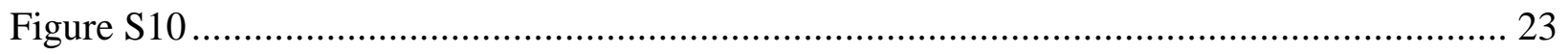

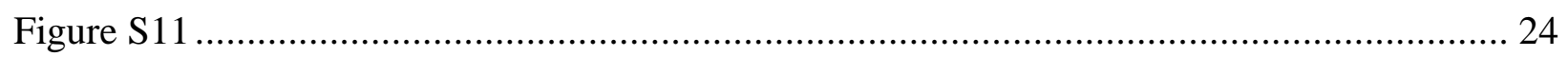

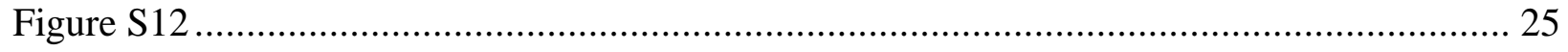

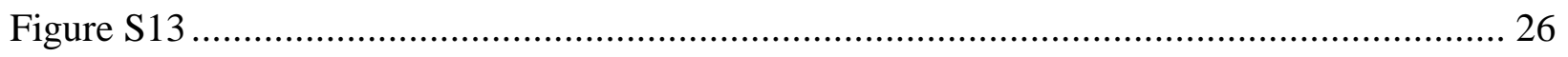

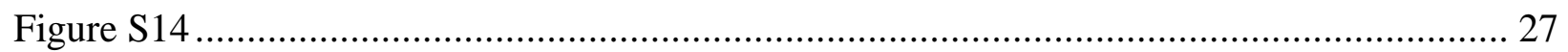

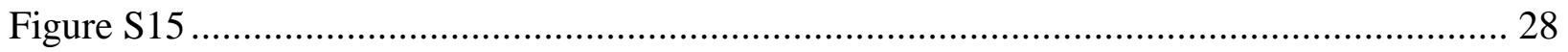

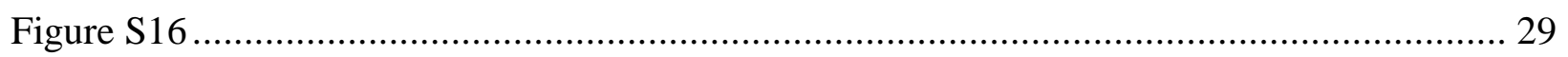

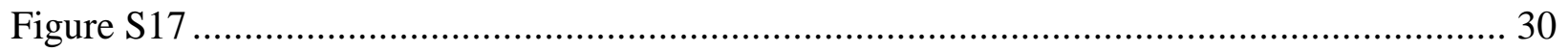

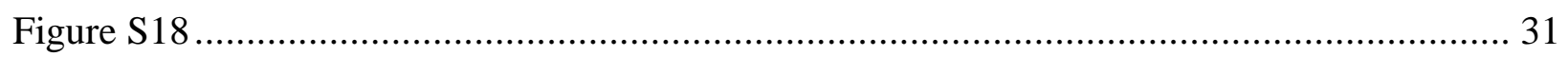

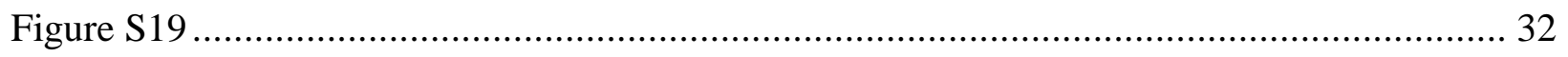

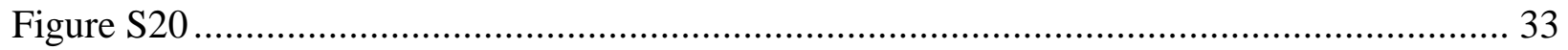

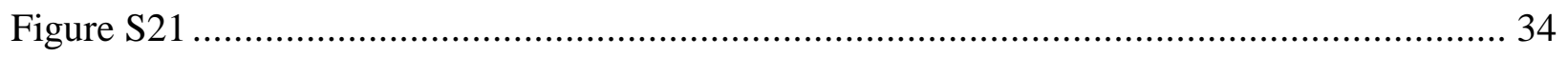

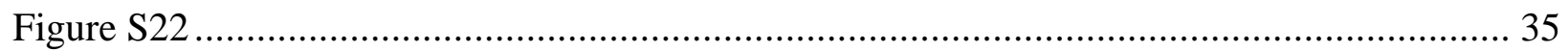

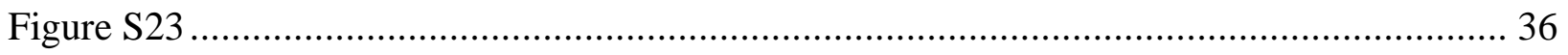


Table S1

Table S2

38

Reference

41 


\section{Materials and methods}

\section{Materials}

Chemicals (e.g., copper (II) acetate and sodium acetate) and solvents (e.g., N,N-Dimethylformamide (DMF) and chloroform) were purchased from PorphyChem and Sigma-Aldrich, and used directly without further purification. Water was purified using a Milli-Q purification system (Merck KGaA). All the reactions were carried out under an ambient atmosphere. Substrates (e.g., $300 \mathrm{~nm} \mathrm{SiO} / \mathrm{Si}$ wafer, quartz glass, and copper grids) were obtained from Plano GmbH.

\section{Methods}

\section{General characterizations.}

Optical microscopy (Zeiss), Atomic force microscopy (AFM) (NT-MDT), transmission electron microscopy (TEM, Zeiss, Libra $200 \mathrm{KV}$ ), and scanning electron microscopy (SEM, Zeiss Gemini 500) equipped with EDX was used to investigate the morphology and structure of the samples. Twodimensional metal-organic frameworks (2D MOFs) films were deposited on Si substrate for SEM, and copper grids for TEM characterizations. All optical microscopy and AFM images were recorded on a 300-nm $\mathrm{SiO}_{2} / \mathrm{Si}$ substrate. UV-visible spectra were measured on a UV-vis-NIR Spectrophotometer Cary 5000 at room temperature using a $3 \times 3 \mathrm{~cm}$ quartz wafer. FTIR spectra were collected using Tensor II (Bruker) with an attenuated total reflection (ATR) unit. The samples were prepared by depositing the films onto a copper foil. X-ray photoelectron spectroscopy (XPS) measurements were carried out using an AXIS Ultra DLD system. Both survey and high-resolution spectra were collected using a beam diameter of $100 \mu \mathrm{m}$. All displayed binding energy values are calibrated to the graphitic C1s peak with a value of $284.6 \mathrm{eV}$.

\section{AC-HRTEM measurements.}

AC-HRTEM imaging and selected area electron diffraction (SAED) were conducted on an image-side $C_{\mathrm{s}}$-corrected FEI Titan 80-300 microscope operated at $300 \mathrm{kV}$. The low-dose technique was applied to preserve the pristine structure of the 2D $c$-MOFs. The electron dose rate for AC-HRTEM and SAED were as low as $200 \mathrm{e}^{-} \AA^{-2} \mathrm{~S}^{-1}$ and $0.15 \mathrm{e}^{-} \AA^{-2} \mathrm{~s}^{-1}$, respectively. For AC-HRTEM imaging, the spherical aberration coefficient $C_{\mathrm{s}}$ was tuned to approx. $-15 \mu \mathrm{m}$ and the images were acquired using over-focus conditions. To maximize the contrast transfer for low spatial frequencies, relative large defocus values $(20-200 \mathrm{~nm})$ were selected.

\section{GIWAXS measurements.}

The GIWAXS measurements were performed at the XRD1 beamline at ELETTRA, Trieste, Italy. The detector was a Dectris Pilatus $2 \mathrm{M}$ and the photon beam energy was $12.398 \mathrm{keV}(\lambda=1 \AA)$. The sampleto-detector distance was $350.2 \mathrm{~mm}$ and was verified using a lanthanhexaborid (LaB6) calibration standard. 
The grazing incidence angle was chosen to be $0.13^{\circ}$ and the sample exposure time was $180 \mathrm{~s}$. The scattering data were then analyzed with WxDiff.

\section{Modeling and electronic structure.}

DFT calculations were carried out using the Vienna ab-initio Simulation Package (VASP) ${ }^{1}$ version 5.4.1. The electronic wave-functions were expanded in a plane-wave basis set with a kinetic energy cutoff of $400 \mathrm{eV}$. The energy stopping criterion was set to EDIFF $=1 \mathrm{E}-6 \mathrm{eV}$. The lattice constants for the monolayer $\mathrm{Cu}_{2}\left[\mathrm{PcCu}-\mathrm{O}_{8}\right]$ was optimized with PBE-D2 and SCAN functionals respectively. Both functionals fall to the local minima at difference of $0.04 \mathrm{~A}$. The PBE-D2 predicts the minima at $\mathrm{a}=\mathrm{b}=18.36$ A, while SCAN at 18.40 A (0.2\% discrepancy in a and b lattice vectors). In the case of MOFs, HSE06, known as one of the most accurate hybrid XC-functional for band gap calculations, is more than 100 times slower than one of the best local functionals HLE17 for band gaps in MOFs. ${ }^{2}$ Unfortunately, using of HLE17 needs some code modifications and recompilation of VASP. According to the recent research in ref. ${ }^{2}$ the mean unsigned error of PBE+U, HSE06 and HLE17 are 0.49, 0.32, and $0.35 \mathrm{eV}$, respectively over 32 semiconductors including MOFs. Thus we conclude that $\mathrm{PBE}+\mathrm{U}$ still remains the best compromise for band gap accuracy concerning the feasibility of these calculations. The geometry optimization convergence was set to forces acting on the ions were smaller than $0.03 \mathrm{eV} \mathrm{A}^{-1}$. Electronion interactions were described using the projector augmented wave (PAW) method. ${ }^{3}$ Generalized gradient approximation (GGA) ${ }^{4}$ of the exchange-correlation energy in the form of Perdew-BurkeErnzerhof (PBE) was applied. ${ }^{5}$ We used DFT+U approach to describe the localized d-orbitals of $\mathrm{Cu}$ ions. The effective Coulomb (U) and exchange (J) terms were set to 4 and $1 \mathrm{eV}$, respectively. ${ }^{6}$ Such a combination of $\mathrm{U}$ and $\mathrm{J}$ are already successfully applied for very similar systems. ${ }^{7}$ The $\mathrm{Cu}_{2}\left[\mathrm{PcCu}-\mathrm{O}_{8}\right]$ monolayer was modeled by adding a large vacuum space, $10 \AA$, in the direction normal to the monolayer. Monkhorst-Pack Gamma-centered grid $^{8}$ with $3 \times 3 \times 1$ dimension was used for K-point sampling of the Brillouin zone for the monolayer during the geometry optimization and $9 \times 9 \times 1$ for band structure calculations. In the computational protocol for the $3 \mathrm{D}$ stacking of $\mathrm{Cu}_{2}\left[\mathrm{PcCu}-\mathrm{O}_{8}\right]$ the $\mathrm{K}$ point grid dimension was changed to $2 \times 2 \times 4$ for the geometry optimization and $4 \times 4 \times 6$ for the band structure calculations and Grimme-D2 correction for the dispersion interlayer interactions was applied. ${ }^{9}$ In order to estimate the possible charge transfer between the layers of $\mathrm{Cu}_{2}\left[\mathrm{PcCu}-\mathrm{O}_{8}\right]$, a Bader analysis was performed. ${ }^{10}$

\section{Variable-temperature conductivity and Hall effect measurements in macroscopic devices for lateral direction.}




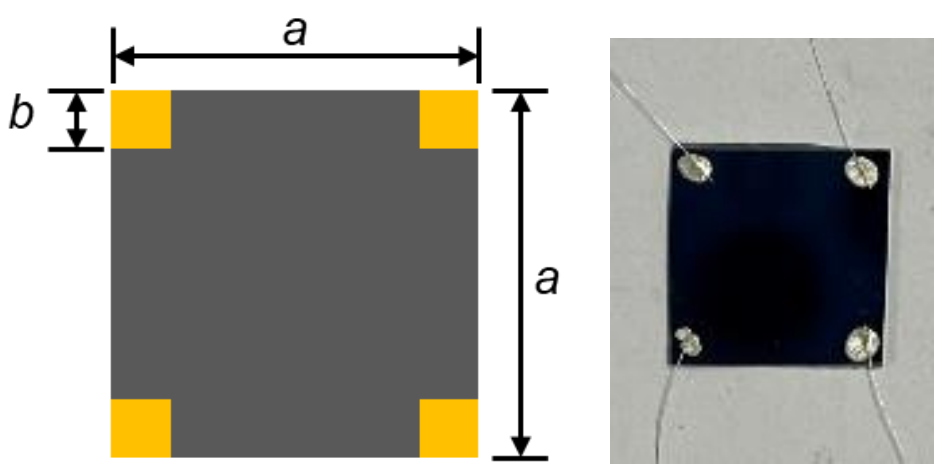

Scheme S1. Van der Pauw sample geometry in square shape. Contacts are orange. $\boldsymbol{a}$ represents the sample size of $1 \mathrm{~cm}$ while $\boldsymbol{b}$ is the contact size of $0.1 \mathrm{~cm}$. Typically, $\boldsymbol{a}$ should be much larger than $\boldsymbol{b}$.

The macroscopic electrical measurements, i.e., temperature-dependent conductivity, carrier concentration, and Hall mobility are characterized in the van der Pauw geometry (Scheme S1) using a commercial Lakeshore Hall System (9700A). This fully integrated Hall measurement system is capable to detect the resistance ranging from $0.04 \mathrm{~m} \Omega$ to $200 \mathrm{G} \Omega$. Moreover, it can set a magnetic field of up to $\pm 9 \mathrm{~T}$ by a superconducting magnet, and the temperature is varied from 1.8 to $400 \mathrm{~K}$ by heating and liquid helium cooling. Silver-conductive glue paste was used to contact the silver wires to the samples. The insulating substrate hereby prevents that shorts are detected even if the silver conductive glue percolates through gaps in the $\mathrm{Cu}_{2}\left[\mathrm{PcCu}-\mathrm{O}_{8}\right]$ films. For the electrical conductivity measurements, samples were measured in the temperature range from $256 \mathrm{~K}$ to $310 \mathrm{~K}$. After confirming the Ohmic contact at different temperatures, we collected an $I-V$ curve by scanning the current from - $10 \mathrm{nA}$ to $10 \mathrm{nA}$ and measuring the voltage at each step at every certain temperature. For the Hall effect measurements, samples were measured at room temperature $(300 \mathrm{~K})$ and a magnetic field perpendicular to the sample plane was swept from -4 T to $4 \mathrm{~T}$. A schematic of a square van der Pauw configuration is shown in Scheme S1. The detailed procedures to carry out resistivity and Hall measurements using the van der Pauw technique are described in the following.

a

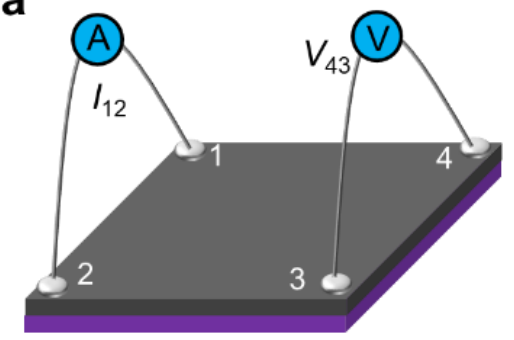

$R_{\mathrm{A}}=\left(R_{43,12}+R_{34,21}+R_{12,43}+R_{21,34}\right) / 4$ b

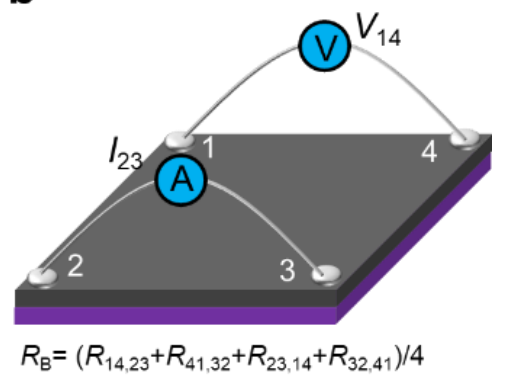

Scheme S2. Schematic view of a van der Pauw configuration used in the determination of the two characteristic resistances $R_{\mathrm{A}}$ and $R_{\mathrm{B} .}{ }^{11-12}$ Contacts are labeled 1, 2, 3, and 4 counterclockwise. 
Resistivity measurements. Van der Pauw method demonstrates that there are two characteristic resistances $R_{\mathrm{A}}$ and $R_{\mathrm{B}}$, associated with the corresponding terminals shown in Scheme $\mathrm{S} 2$. In the basic van der Pauw contact arrangement, the four contacts on the sample are numbered in ascending order. It is important to use the same batch of wire for all four leads to minimizing thermoelectric effects. Similarly, all contacts should consist of the same material.

To obtain the two characteristic resistances, one applies a DC current into contact 1 and out of contact 2 and measures the voltage $V_{43}$ from contact 4 to contact 3 as shown in Scheme S2a. Next, the current $I$ is applied into contact 2 and out of contact 3 while measuring the voltage $V_{14}$ from contact 1 to contact 4 (shown in Scheme S2b). Then, one should reverse the polarity of the current $I_{21}\left(I_{32}\right)$ and measure $V_{34}$ $\left(V_{41}\right)$. After that, the same process will repeat for the remaining voltage values. Eight measurements of voltage yield the following eight values of resistance, all of which must be positive:

$$
\begin{aligned}
& R_{21,34}=\frac{V_{34}}{I_{21}}, R_{12,43}=\frac{V_{43}}{I_{12}}, \\
& R_{32,41}=\frac{V_{41}}{I_{32}}, R_{23,14}=\frac{V_{14}}{I_{23}}, \\
& R_{43,12}=\frac{V_{12}}{I_{43}}, R_{34,32}=\frac{V_{21}}{I_{34}} \\
& R_{14,23}=\frac{V_{23}}{I_{14}}, R_{41,32}=\frac{V_{32}}{I_{41}},
\end{aligned}
$$

Thus, the characteristic resistances $R_{\mathrm{A}}$ and $R_{\mathrm{B}}$ are calculated using the following expressions:

$$
\begin{aligned}
& R_{A}=\frac{R_{43,12}+R_{34,21}+R_{12,43}+R_{21,34}}{4} \\
& R_{B}=\frac{R_{14,23}+R_{41,32}+R_{23,14}+R_{32,41}}{4}
\end{aligned}
$$

The sheet resistance $R_{\mathrm{S}}$ can be determined numerically from the two characteristic resistances $R_{\mathrm{A}}$ and $R_{\mathrm{B}}$ through the van der Pauw equation:

$$
\exp \left(\frac{-\pi R_{A}}{R_{S}}\right)+\exp \left(\frac{-\pi R_{B}}{R_{S}}\right)=1
$$

Therefore, the bulk electrical resistivity $\rho$ can be calculated using

$$
\rho=R_{s} d
$$

where $d$ is the thickness of the $\mathrm{Cu}_{2}\left[\mathrm{PcCu}-\mathrm{O}_{8}\right]$ sample. 


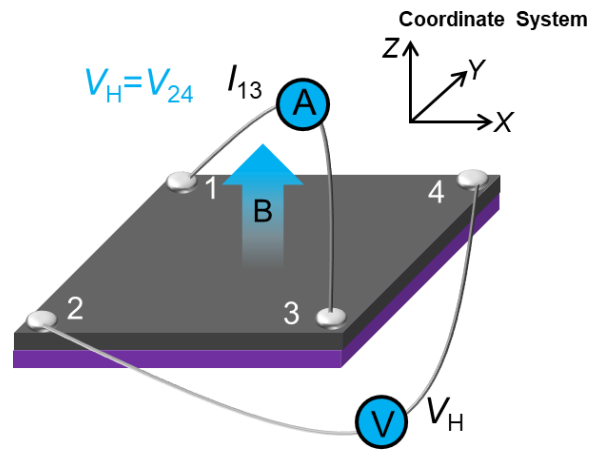

Scheme S3. Schematic of a van der Pauw configuration used in the determination of the Hall voltage $V_{\mathrm{H}}$.

Hall measurements. The objective of the Hall measurement by the van der Pauw technique is to determine the sheet carrier density $\left(n_{S}\right)$ by measuring the Hall voltage $\left(V_{H}\right)$. The Hall voltage measurement consists of a series of voltage measurements with a constant current $I$ and a constant magnetic field $B$ applied perpendicular to the plane of the sample. An example is shown in Scheme S3. To measure the Hall voltage $V_{H}$, a current $I$ is forced through the opposing pair of contacts 1 and 3 and the Hall voltage $V_{H}=V_{24}$ is measured across the remaining pair of contacts 2 and 4 . Once the Hall voltage $V_{H}$ is acquired, the sheet carrier density $n_{S}$ can be calculated via

$$
n_{S}=\frac{I B}{e \gamma V_{H}}
$$

where $I$ is current, $B$ is the magnetic field, $V_{H}$ is the Hall voltage, $e\left(1.602 \times 10^{-19} \mathrm{C}\right)$ is the elementary charge and $\gamma$ is the Hall scattering factor (generally assumed to be 1 in heavily doped Si system) ${ }^{13-14}$. If the effective thickness of the conducting layer $d$ is known, the bulk density $(N)$ can be determined as:

$$
N=\frac{n_{s}}{d}
$$

Besides, the polarity of the Hall voltage indicates which type of carrier dominates the conducting behavior of the sample, and the Hall voltage is negative for $n$-type semiconductors (electron) and positive for $p$ type semiconductors (hole). As described before, the sheet resistance $R_{S}$ of the semiconductor can be conveniently determined by using the van der Pauw resistivity measurement technique. Since sheet resistance involves both sheet density and mobility, the Hall mobility can be obtained via the equation below:

$$
\mu=\frac{\left|V_{H}\right|}{I B R_{S}}=\frac{1}{q n_{S} R_{S}}
$$

Normally, the unit of the Hall mobility is $\mathrm{cm}^{2} \mathrm{~V}^{-1} \mathrm{~s}^{-1}$. 


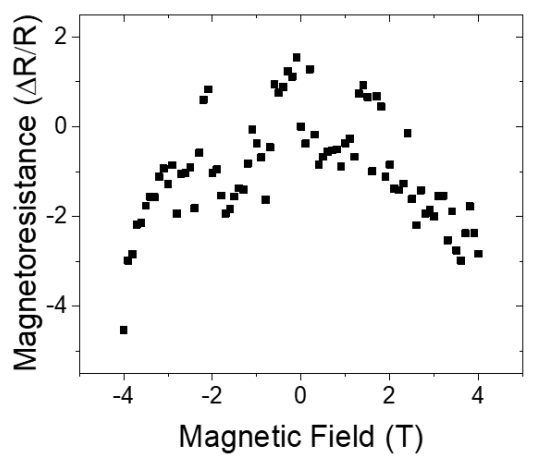

Scheme S4. Magnetoresistance as a function of the magnetic field.

The magnetoresistance as the function of the magnetic field is shown in Scheme S4. The magnetoresistance is in the order of $10^{8} \Omega / \mathrm{sq}$, and the line shape is relatively symmetric. Therefore, the influence of magnetoresistance on Hall resistance can be neglected.

\section{Macroscopic vertical devices.}

For the vertical devices as shown in Figure $3 \mathrm{a}$ (right), gold contacts with sizes of $350 \mu \mathrm{m}$ were evaporated through shadow masks, resulting in the structure shown in Figure S18. The channel length $(L)$ is the thickness of the sample, which was varied between 20 and $250 \mathrm{~nm}$, and the channel area $(A)$ is $\sim 1.3 \times 10^{-}$ ${ }^{3} \mathrm{~cm}^{2}$. The conductivity was calculated with the equation:

$$
\sigma=\frac{1}{R_{\text {sample }}} \cdot \frac{L}{A}
$$

where $R_{\text {sample }}=V / I$.

We calculated the conductivity in Figure $3 \mathrm{~d}$ by $R=\left(\frac{\partial I}{\partial V}\right)^{-1}$ and the conductivity using $\sigma=\rho^{-1}=$ $\left(R \cdot \frac{A}{L}\right)^{-1}$ with $A, L$, the channel area, and length, respectively.

The values of conductivity as presented in Figure $3 \mathrm{~d}$ and Figure $3 \mathrm{e}$ were calculated after excluding the contact resistance $\left(2 R_{\mathrm{c}}\right)$.

$$
R_{\text {sample }}=R_{\text {total }}-2 R_{\mathrm{c}}
$$

where $R_{\text {total }}$ was resolved by linear fitting the $I-V$ curve in the voltage range from -1 to $1 \mathrm{~V}$; and $2 R_{\mathrm{c}}$ was extracted with the use of transmission line measurement (TLM) to be $\sim 2600 \Omega$.cm in vertical devices.

\section{Local characterization of directional charge transport.}

Lateral transport. Field-effect transistors (FETs) for local lateral transport measurements were fabricated on substrates of highly doped $\mathrm{Si}$ with $300 \mathrm{~nm} \mathrm{SiO}$. Hexagonal boron nitride ( $h$-BN) flakes were exfoliated and the $\mathrm{Cu}_{2}\left[\mathrm{PcCu}-\mathrm{O}_{8}\right]$ film was applied on top, as described in the main text. Suitable flakes were located using an optical microscope (Zeiss) and electrical contacts were structured using 
electron beam lithography (Raith e-LINE system) in an ultrahigh vacuum. Contacts of $0.5 \mathrm{~nm}$ titanium (at $0.1 \AA^{-1}$ ) and $60 \mathrm{~nm}$ gold (at $1.0 \AA^{-1}$ ) were evaporated in an UHV evaporation chamber (BesTec) at $\sim 10^{-7}$ mbar. In this manner, transistor geometries with several contacts per flake were obtained (e.g. six in Figure S19). By detecting $I$ - $V$ curves for each pair of neighboring contacts, in sum 15 measurements were taken.

The electrical measurements were taken in a LakeShore Probe station CRX-VF at room temperature and $3 \times 10^{-5}$ mbar using two Keithley Sourcemeters 2450 . We detected no field effect, presumably due to a significant amount of charge trapping in the thin film, even by applying gate voltages up to $\pm 120 \mathrm{~V}$. However, the use of $h$-BN was observed to increase the detected current in twopoint measurements and the stability against degradation, compared to $h$-BN free substrates. Due to its two-dimensional nature, the roughness of the underneath lying substrate is minimized, and trapping at the insulator- $\mathrm{Cu}_{2}\left[\mathrm{PcCu}-\mathrm{O}_{8}\right]$-interface is suppressed.

From the detected currents, we calculated the resistance by $R=\left(\frac{\partial I}{\partial V}\right)^{-1}$ and the conductivity using $\sigma=\rho^{-1}=\left(R \cdot \frac{W d}{L}\right)^{-1}$ with $W, L$, the channel width and length, respectively. The film thickness, d, was measured by the step height at a scratch in the film down to the substrate using a Bruker Icon AFM in tapping mode, exemplarily shown for one device in Scheme S5.

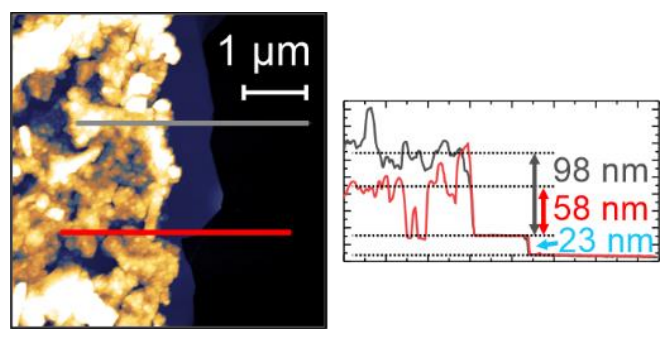

Scheme S5. Measurement of mean film thickness at an arbitrarily chosen spot by AFM step height down to the substrate. Red and grey denote the detected film height for the $\mathrm{Cu}_{2}\left[\mathrm{PcCu}-\mathrm{O}_{8}\right]$ film at two different positions, blue is the height of the $h$-BN.

Besides the conductivity, the total resistance $\mathrm{R}$ was plotted for increasing channel lengths $\mathrm{L}$ to estimate the contact resistance of the nanoscopic gold contacts. However, no R(L) dependence could be observed. This might be drawn back to the inhomogeneity of the $\mathrm{Cu}_{2}\left[\mathrm{PcCu}-\mathrm{O}_{8}\right]$ film within the size of one $h$-BN flake.

Vertical transport. For the local electrical measurements in the vertical direction, $\mathrm{Cu}_{2}\left[\mathrm{PcCu}-\mathrm{O}_{8}\right]$ films were applied on substrates with a $50 \mathrm{~nm}$ thick layer of silver. c-AFM measurements were taken, as it was observed before, that the evaporation of gold on very thin films of $\mathrm{Cu}_{2}\left[\mathrm{PcCu}-\mathrm{O}_{8}\right]$ film on top of conductive substrates may lead to shorts, due to percolating contact material or because the 
evaporation of gold destroys the $\mathrm{Cu}_{2}\left[\mathrm{PcCu}-\mathrm{O}_{8}\right]$ film. The measurements were taken using the c-AFM mode of an Icon AFM (Bruker) at ambient pressure and room temperature. The tip used is an SCMPIT-V2 ( $k=2.8 \mathrm{~N} \mathrm{~m}^{-1}$, PtIr coating), at which we applied voltages between 1 and $4 \mathrm{~V}$, while keeping the silver layer grounded. The contact area between tip and film is estimated to $100 \mathrm{~nm}^{2} .{ }^{15}$ This measurement setup corresponds to the suggested setup by Jiang, Weber, et al. to prevent local anodic oxidation, and we can exclude degradation of the tip due to the small number of scans we have made (ten in total for each of two different positions on the film). ${ }^{15}$ To calculate the local conductivity of the film in the intralayer direction, we extracted the absolute current values at the highest points within one scan (compare Figure S20) for the reasons given in the main text and below. The resulting plots for the first four points are presented in Scheme S6a. From fitting a linear curve for each data set, the resistivity and conductivity are calculated as presented for the lateral transport measurements (with $\mathrm{R}=1 / \mathrm{slope}$ and $\mathrm{wd}=\mathrm{A}=100 \mathrm{~nm}^{2}$ ).
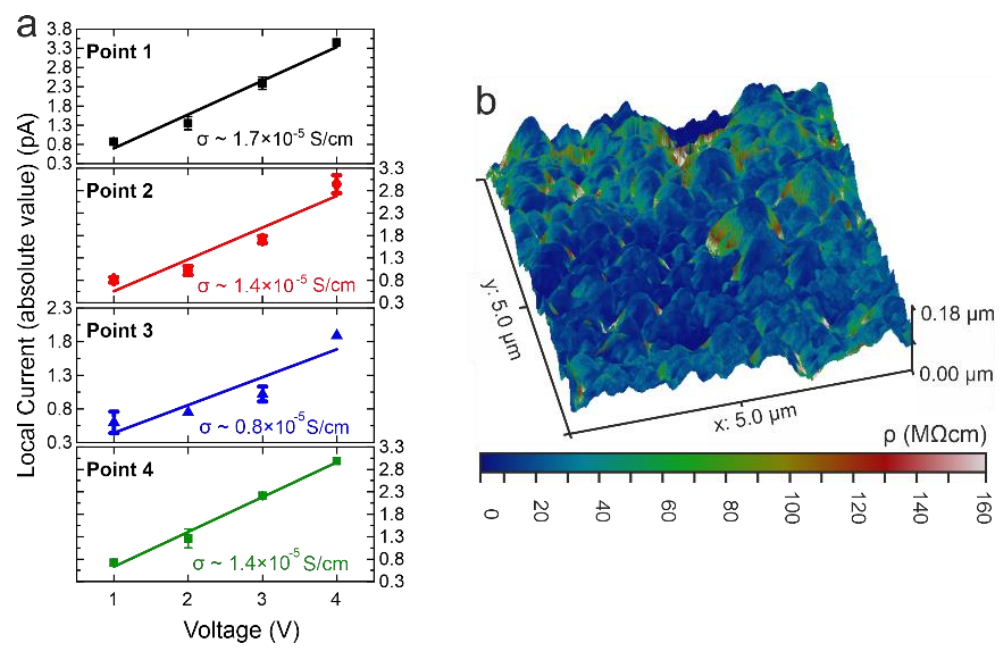

Scheme S6. (a) Extracted currents from the four highest points detected in Figure S20a with a linear fit and the resulting conductivity, calculated as explained in the text. (b) 3D topography plot of the spot shown in Figure S20c with the local resistivity in the color scale.

For the calculation of the film thickness, we determined the mean thickness of the $\mathrm{Cu}_{2}\left[\mathrm{PcCu}-\mathrm{O}_{8}\right]$ film to $d=19 \pm 3 \mathrm{~nm}$ by measuring the step height to the substrate, as shown in Scheme S7. However, this value cannot be used for local resistivity/conductivity calculations, as both topography scans at the two arbitrarily chosen spots (Figure S20) show that the film height varies by about 90 to $160 \mathrm{~nm}$. Hence, the detected height of a certain position with respect to the lowest point within one scan is taken as effective film thickness $d_{\text {eff. }}$ By only analyzing the highest points, the error according to the unknown underneath lying film thickness will thereby be reduced. 


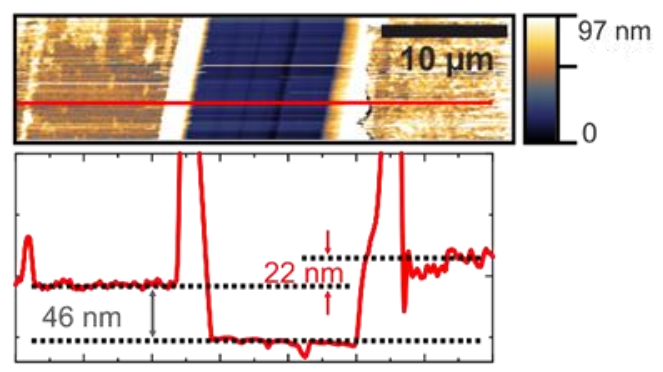

Scheme S7. Measurement of mean film thickness at an arbitrarily chosen spot by AFM step height down to the substrate. In red, the average thickness of the $\mathrm{Cu}_{2}\left[\mathrm{PcCu}-\mathrm{O}_{8}\right]$ film is given, grey denotes the thickness of the silver layer.

Another way to calculate the resistivity without the use of a fit function is given in Scheme S6b. Here, we directly calculated and plotted the resistivity $\rho=\frac{4 V}{I(x, y)} \cdot \frac{A_{e f f}}{d_{e f f}(x, y)}$ from the c-AFM plot taken at $V_{t}=4 \mathrm{~V}$ and the topography scan using the arithmetic function of Gwydion. ${ }^{16}$ We did this to prove if the deviation of the $I-V$-dependence from the Ohmic law has a high impact on the resistivity values. From the three dimensional (3D) plot correlating the topography (z-axis) and resistivity (color scale), we can conclude that: (1) the resistivity is mostly independent of the film height; (2) at steep flanks the resistivity takes the highest values, presumably due to bending of the AFM tip or decreased contact between tip and sample (the resistivity values were consequently extracted at positions of flat geometry), and (3) the resistivity values calculated from the I-V dependence as explained above tend to be underestimated (and therefore the conductivities in the main text are overestimated), but the results go along with the previously made observation, that the resistivity of the $\mathrm{Cu}_{2}\left[\mathrm{PcCu}_{2}-\mathrm{O}_{8}\right]$ film along the intralayer direction is much higher than along interlayer direction.

\section{Synthetic Procedures}

\section{Synthesis of PcM-OHs}

PcCu-OMes: The synthesis was adopted from reported literature procedures. ${ }^{17-19}$ 1, 2-dibromo-4, 5dimethoxybenzene $(8.87 \mathrm{~g}, 0.03 \mathrm{~mol})$, and $8.06 \mathrm{~g}(0.09 \mathrm{~mol})$ of $\mathrm{CuCN}$ was refluxed in $\mathrm{DMF}$ at $165^{\circ} \mathrm{C}$ for $5 \mathrm{~h}$. After cooling at room temperature, $300 \mathrm{ml}$ of concentrated ammonium hydroxide was added into the above reaction mixture and it was stirred at room temperature for $12 \mathrm{~h}$ under air. Then the green solid was obtained by filtration and washed with a copious amount of diluted ammonium hydroxide and water. The dry, crude olive-green product was placed in the thimble of a Soxhlet extractor and extracted with methanol and acetone for 1 day. Then, the green solid was collected.

PcCu-OH8: 2 g as-prepared PcCu-OMe was dissolved into $300 \mathrm{ml}$ of dry dichloromethane, and boron tribromide $(4.8 \mathrm{ml})$ was added under Ar atmosphere. After stirring at room temperature for $2 \mathrm{~h}$, the mixture was refluxed for $20 \mathrm{~h}$. After cooling down, $50 \mathrm{ml}$ of methanol was slowly added to the mixture. 
Then the precipitates were filtered and washed with $\mathrm{MeOH}$, water, and dichloromethane, centrifuged many times with water, $\mathrm{MeOH}$, and dichloromethane until the supernatant became almost transparent, as well as dried under vacuum.

PcFe-OH8: $\mathrm{PcCu}_{-} \mathrm{OH}_{8}(0.005 \mathrm{mmol})$ was well dispersed into DMF $(25 \mathrm{ml})$. After adding $2.2 \mathrm{ml}$ of $\mathrm{NH}_{3} \cdot \mathrm{H}_{2} \mathrm{O}$ (v/v: $50 \%$ ), the $\mathrm{PcCu}-\mathrm{OH}_{8}$ solution was diluted with $30 \mathrm{ml}$ of $\mathrm{H}_{2} \mathrm{O}$. After sonication for $5 \mathrm{~min}$, $5 \mathrm{ml}$ of $\mathrm{Fe}(\mathrm{acac})_{2}(0.01 \mathrm{mmol})$ solution was added to the above mixture. This mixture was transferred into the autoclave and heated at $120{ }^{\circ} \mathrm{C}$ for $40 \mathrm{~h}$. Finally, the solid was obtained by filtration, washed with DMF, $\mathrm{H}_{2} \mathrm{O}$, and acetone, and vacuum dried at room temperature.

Reagents: All solvents, reagents, and chemicals were purchased from commercial suppliers (SigmaAldrich, TCI, and abcr GmbH.) and used without further purification unless specially addressed. All the reactions were performed using the standard vacuum-line and Schleck techniques under argon or vacuum.

Synthesis of $\mathrm{Cu}_{2}\left[\mathbf{P c C u}-\mathrm{O}_{8}\right]$ MOF film. $\mathrm{PcCu}_{-} \mathrm{OH}_{8}(1 \mathrm{mg})$ was dissolved in $3 \mathrm{ml}$ of mixed chloroform/DMF solvent $(2: 1$, in volume $)$. A beaker $(80 \mathrm{ml}$, diameter $=6 \mathrm{~cm})$ was filled with $40 \mathrm{ml} \mathrm{Milli-}$ Q water under the ambient condition to form a static air/water interface. $200 \mu \mathrm{l}$ of a freshly prepared $\mathrm{PcCu}-\mathrm{OH}_{8}$ solution was carefully spread drop-by-drop onto the water surface with a syringe. The solvent was allowed to evaporate for 30 minutes. Then, $5 \mathrm{ml}$ copper(II) acetate $\left(1 \mathrm{mg} \mathrm{ml}^{-1}\right)$ and $2 \mathrm{ml}$ sodium acetate $\left(1 \mathrm{mg} \mathrm{ml}^{-1}\right)$ were subsequently added to the subphase. The reaction system was left undisturbed under an ambient atmosphere for $18 \mathrm{~h}$ to obtain a film on the water surface. The asprepared film was then transferred onto substrates.

Synthesis of $\mathrm{Cu}_{2}\left[\mathrm{PcFe}-\mathrm{O}_{8}\right]$ MOF film. $\mathrm{PcFe}_{8}-\mathrm{OH}_{8}(1 \mathrm{mg})$ was dissolved in $3 \mathrm{ml}$ of mixed chloroform/DMF solvent $(2: 1$, in volume). A beaker $(80 \mathrm{ml}$, diameter $=6 \mathrm{~cm})$ was filled with $40 \mathrm{ml}$ MilliQ water under the ambient condition to form a static air/water interface. $200 \mu 1$ of a freshly prepared $\mathrm{PcCu}-\mathrm{OH}_{8}$ solution was carefully spread drop-by-drop onto the water surface with a syringe. The solvent was allowed to evaporate for 30 minutes. Then, $5 \mathrm{ml}$ copper(II) acetate $\left(1 \mathrm{mg} \mathrm{ml}^{-1}\right)$ and $2 \mathrm{ml}$ sodium acetate $\left(1 \mathrm{mg} \mathrm{ml}^{-1}\right)$ were subsequently added to the subphase. The reaction system was left undisturbed under an ambient atmosphere for $18 \mathrm{~h}$ to obtain a film on the water surface. The asprepared film was then transferred onto substrates. 


\section{Supporting Figures and Tables}

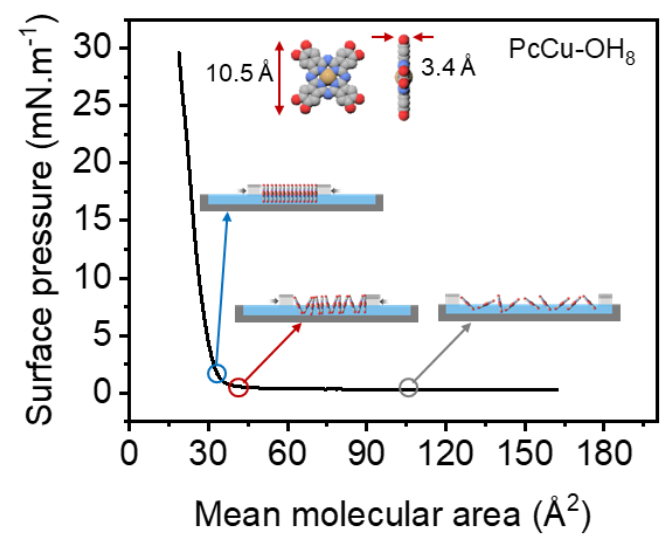

Figure S1. Surface pressure-mean molecular area $(\pi-\mathrm{A})$ isotherm for $\mathrm{PcCu}-\mathrm{OH}_{8} .50 \mu \mathrm{l}$ freshly prepared solution of $\mathrm{PcCu}-\mathrm{OH}_{8}(0.47 \mathrm{mM})$ in chloroform/DMF (2:1 in volume) was spread onto the water surface. After 10 min evaporation of the solvent, the Delrin barriers were driven forward to compress $\mathrm{PcCu}-\mathrm{OH}_{8}$ on the water surface at a rate of $5 \mathrm{~mm} \mathrm{~min}^{-1}$ until the surface pressure reaching $30 \mathrm{mN} \mathrm{m}^{-1}$. The small molecular area implies that the $\mathrm{PcCu}-\mathrm{OH}_{8}$ molecules stand vertically on the water surface. 

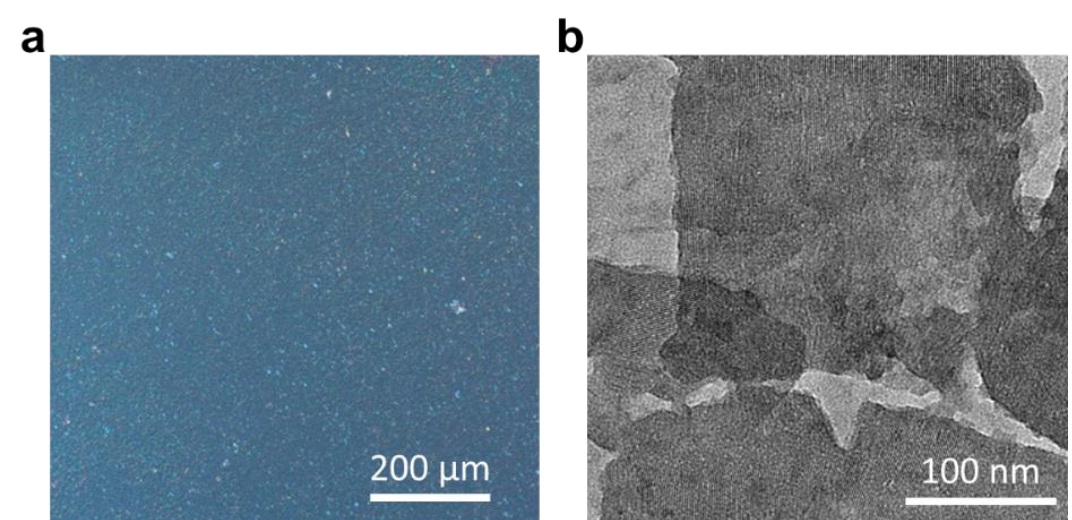

Figure S2. Structural characterization of the $\mathrm{PcCu}-\mathrm{OH}_{8}$ ligand self-assembly. Optical microscopy (OM) (a) and (b) TEM images of the self-assembly $\mathrm{PcCu}-\mathrm{OH}_{8}$. 
Figure S3. OM image of $\mathrm{Cu}_{2}\left[\mathrm{PcCu}-\mathrm{O}_{8}\right]$ film. 


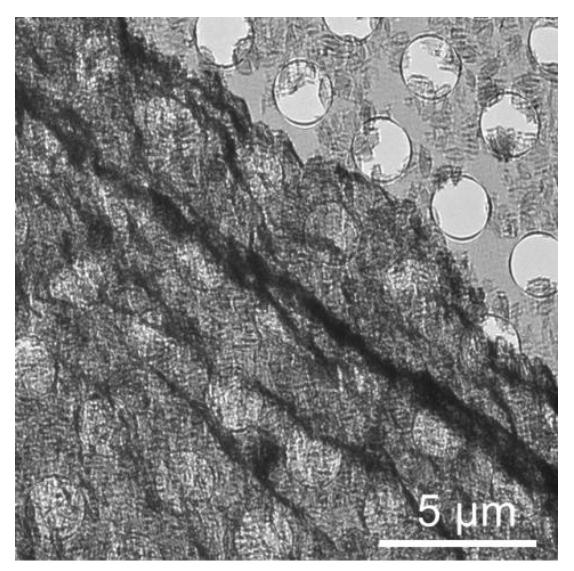

Figure S4. TEM image of $\mathrm{Cu}_{2}\left[\mathrm{PcCu}-\mathrm{O}_{8}\right]$ film. 

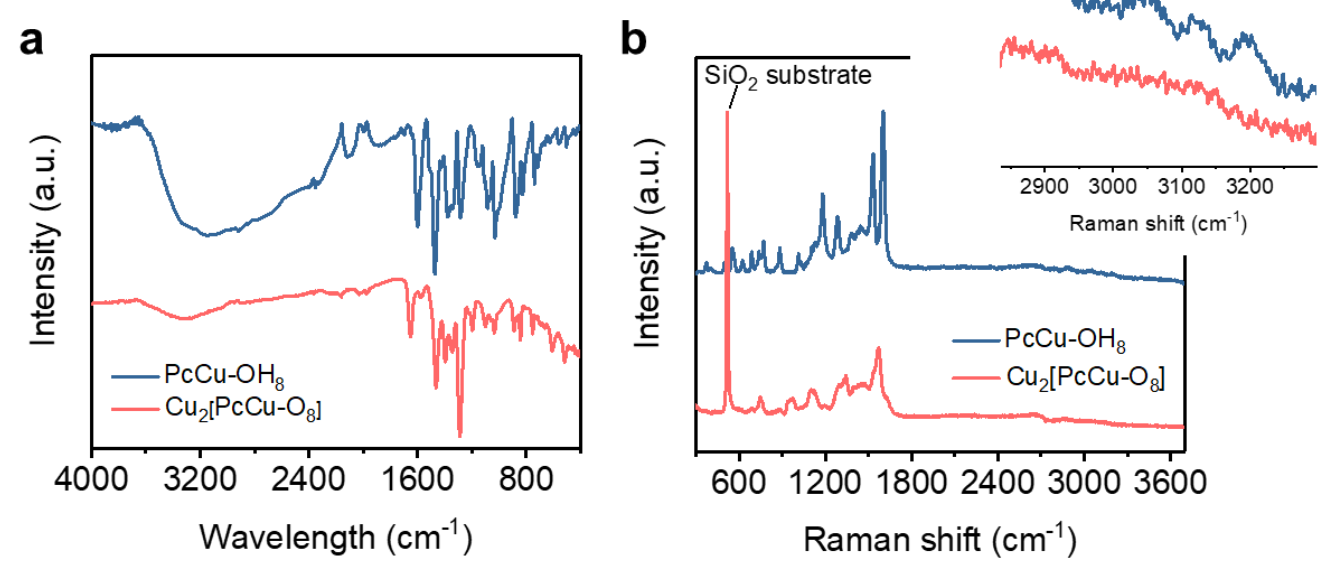

Figure S5. ATR-FTIR (a) and Raman (b) spectra of $\mathrm{PcCu}-\mathrm{OH}_{8}$ monomer and $\mathrm{Cu}_{2}\left[\mathrm{PcCu}-\mathrm{O}_{8}\right]$. 

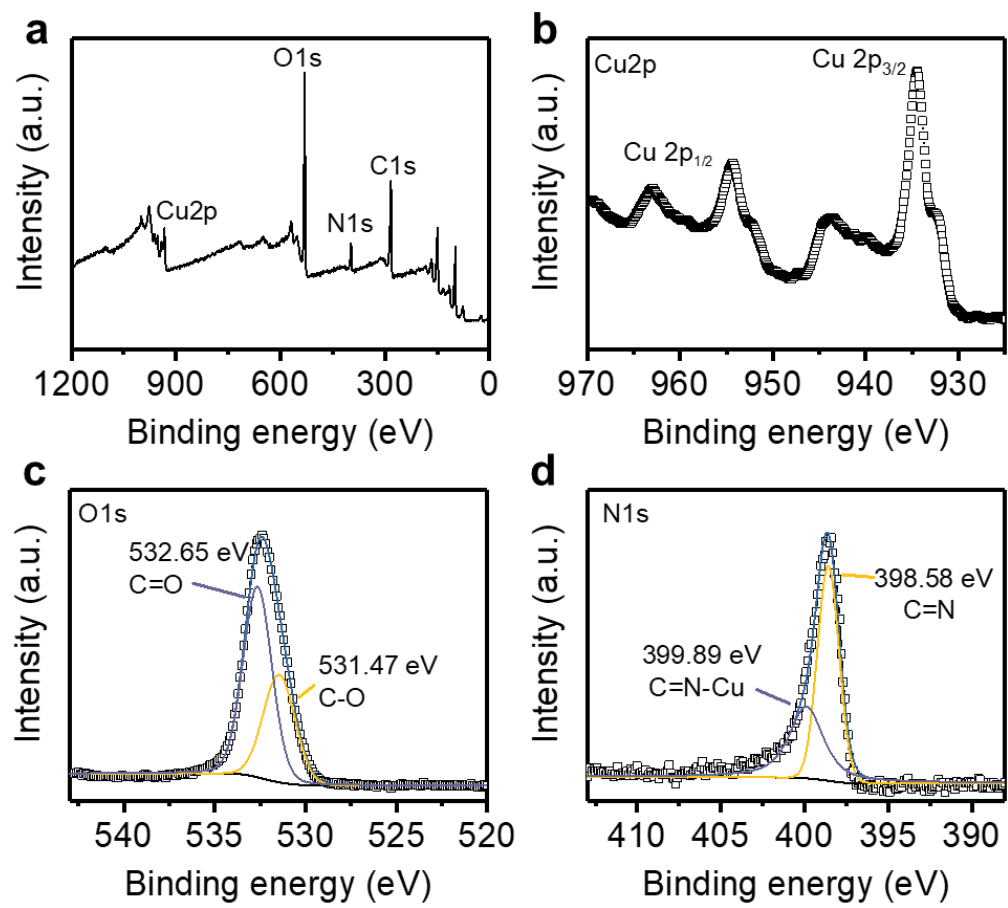

Figure S6. Chemical structure analysis. Survey (a) $\mathrm{Cu} 2 \mathrm{p}(\mathrm{b}), \mathrm{O} 1 \mathrm{~s}(\mathrm{c})$ and $\mathrm{N} 1 \mathrm{~s}$ (d) XPS spectra of $\mathrm{Cu}_{2}\left[\mathrm{PcCu}-\mathrm{O}_{8}\right]$. 


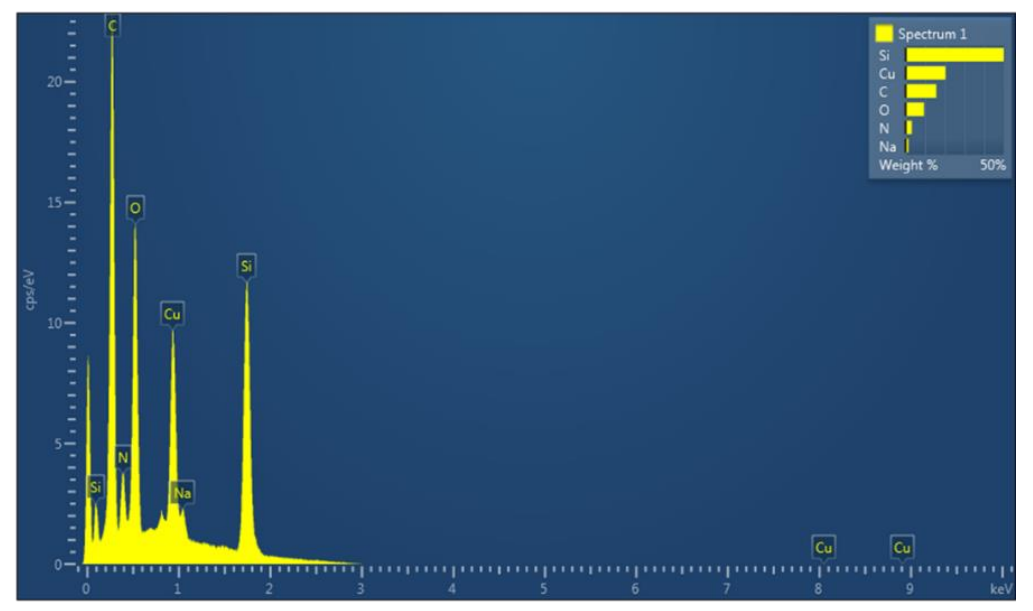

Figure S7. EDS spectrum revealing the sheet composition with respect to $\mathrm{C}, \mathrm{N}, \mathrm{O}, \mathrm{Cu}$ and $\mathrm{Na}$. 
a

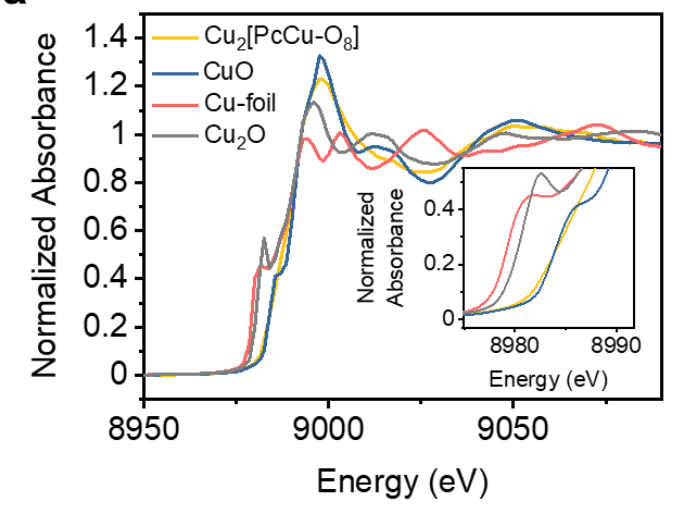

b

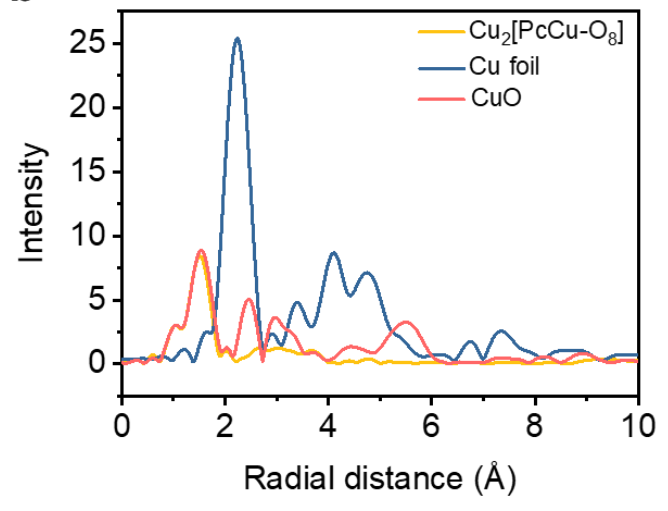

Figure S8. (a) Normalized $\mathrm{Cu}$ K-edge XANES spectra of $\mathrm{Cu}_{2}\left[\mathrm{PcCu}-\mathrm{O}_{8}\right], \mathrm{CuO}, \mathrm{Cu}$ foil and $\mathrm{Cu}_{2} \mathrm{O}$. (b) Fourier transformation EXAFS spectra at $\mathrm{Cu}$ K-edge of $\mathrm{Cu}_{2}\left[\mathrm{PcCu}-\mathrm{O}_{8}\right]$ with $\mathrm{Cu}$ foil and $\mathrm{CuO}$ as contrast. 


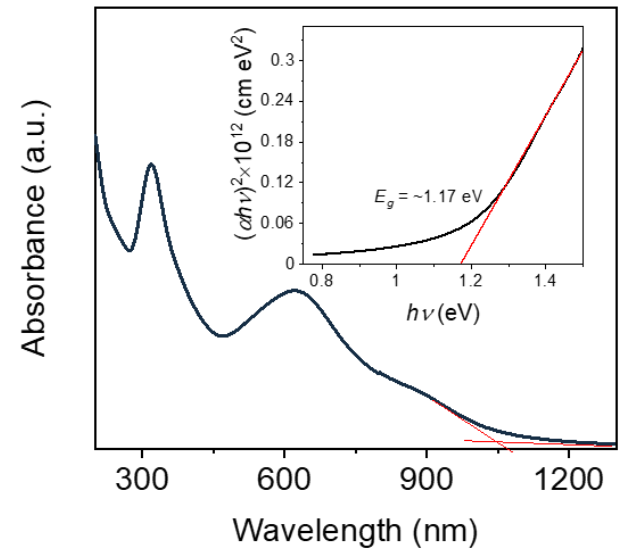

Figure S9. The UV-vis spectrum of $\mathrm{Cu}_{2}\left[\mathrm{PcCu}-\mathrm{O}_{8}\right]$. Inset: the Tauc plot indicates a direct bandgap of $\sim 1.17 \mathrm{eV}$ for $\mathrm{Cu}_{2}\left[\mathrm{PcCu}-\mathrm{O}_{8}\right]$ film. 


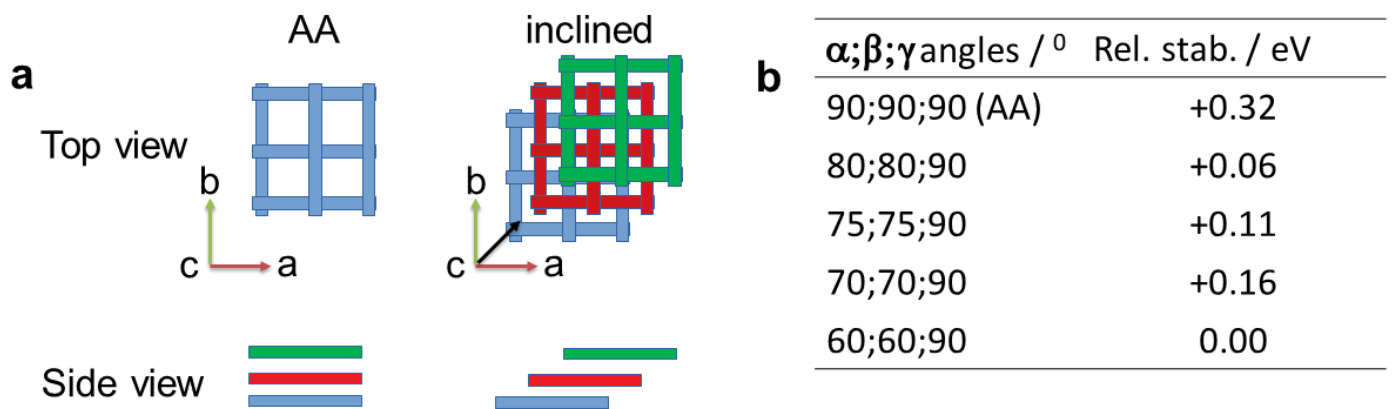
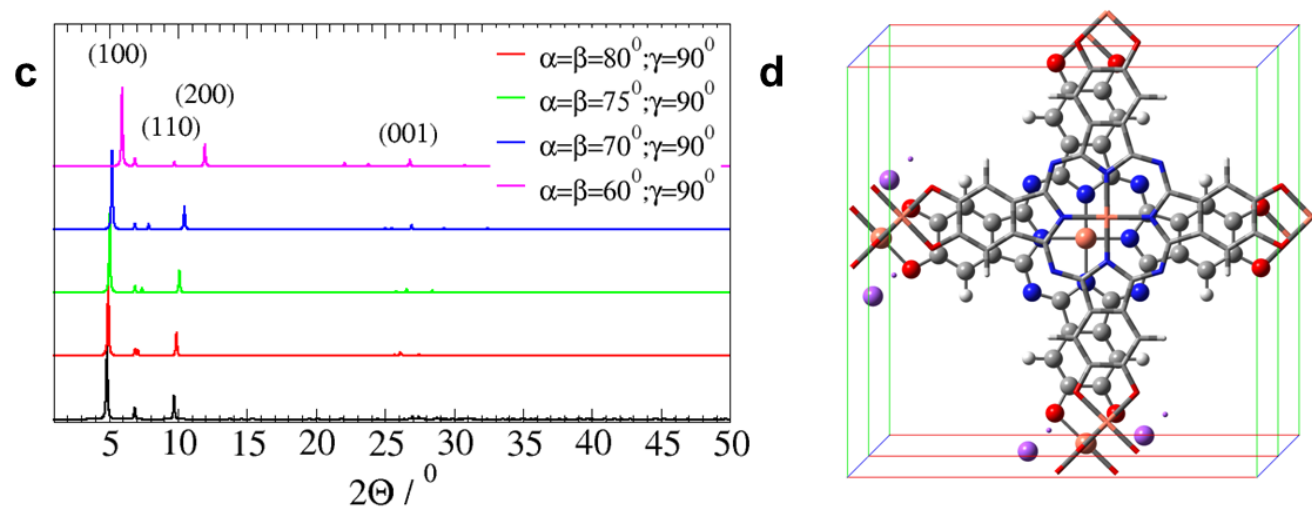

Figure S10. (a) Schematic representation of transformation from AA to inclined stacking mode. (b) Relative stability for different stacking orders starting from AA toward inclined stacking. The inclination of the structure was achieved by changing $\alpha$ and $\beta$ angles from $90^{\circ}$ to $60^{\circ}$ of the unit cell, at a constant interlayer distance of $0.33 \mathrm{~nm}$. The relative stability of different stacking orders starting from AA towards inclined stacking was calculated and the corresponding numbers are collected. (c) Simulated PXRD for several different structures of AA $\rightarrow$ inclined stacking transformation. Note that the stronger (200) peak in the GIWAXS profile might be due to the edge-on orientation of the $\mathrm{Cu}_{2}\left[\mathrm{PcCu}-\mathrm{O}_{8}\right]$ film, and the presence of an unresolved angle (should be below 90 ) between the basal plane and the substrate. (d) Atomistic model of the inclined structure with $\alpha=\beta=75^{\circ}$. Orange, grey, red, blue, and purple spheres represent $\mathrm{Cu}$, $\mathrm{C}, \mathrm{O}, \mathrm{N}$, and $\mathrm{Na}$ atoms, respectively. 

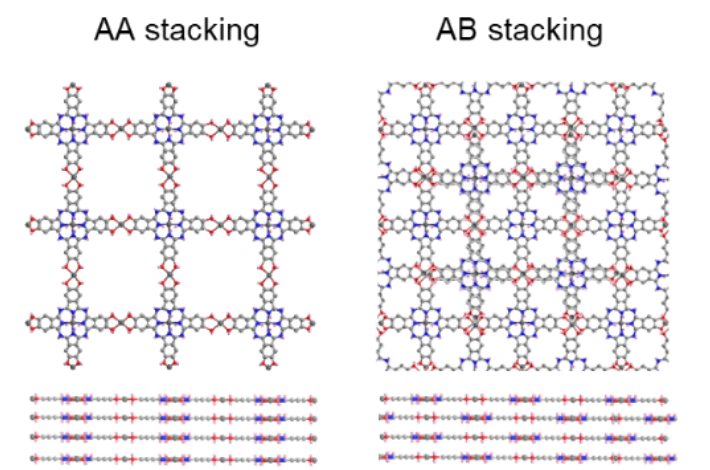

AA-serrated stacking

AA-inclined stacking
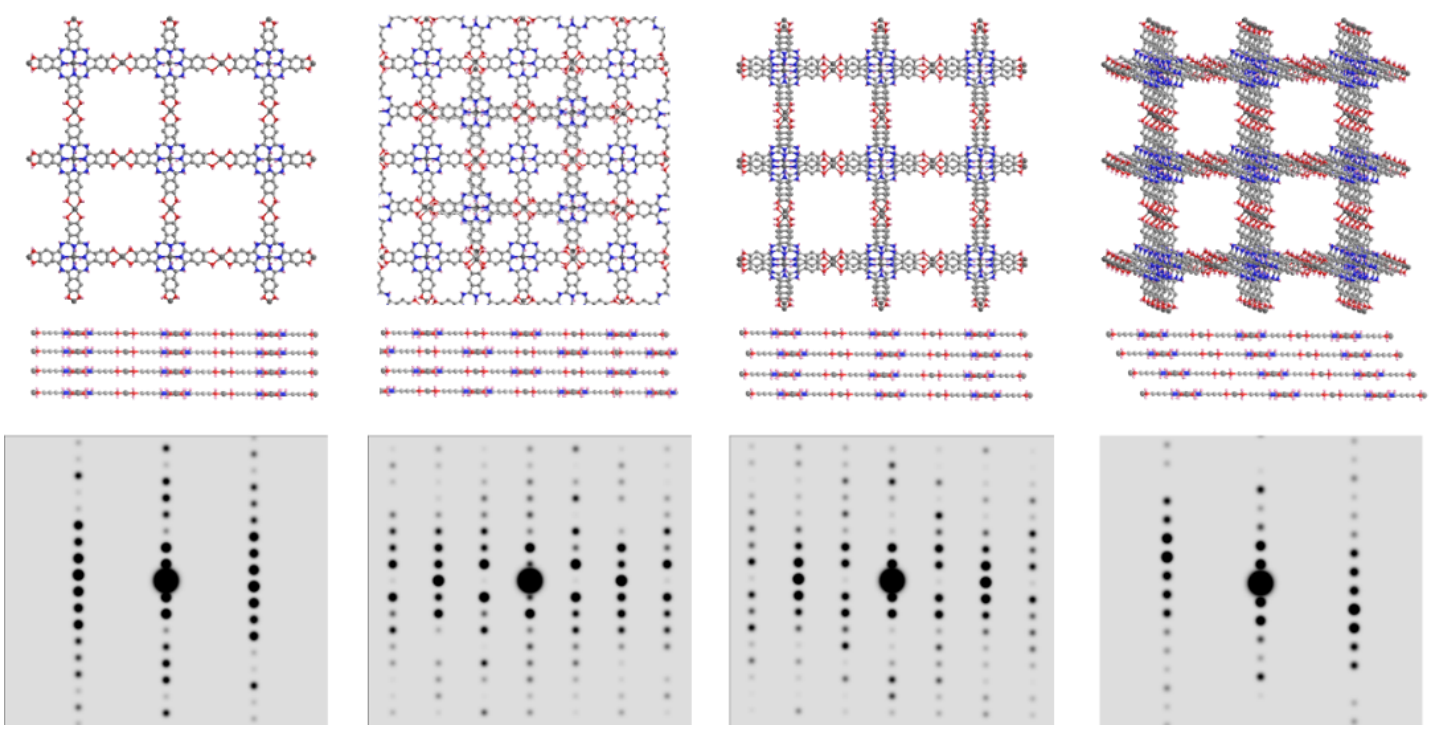

Figure S11 DFT models of different stacking modes of $\mathrm{Cu}_{2}\left[\mathrm{PcCu}-\mathrm{O}_{8}\right]$ ([001] projection) and corresponding simulated SAED patterns ([100] projection). 

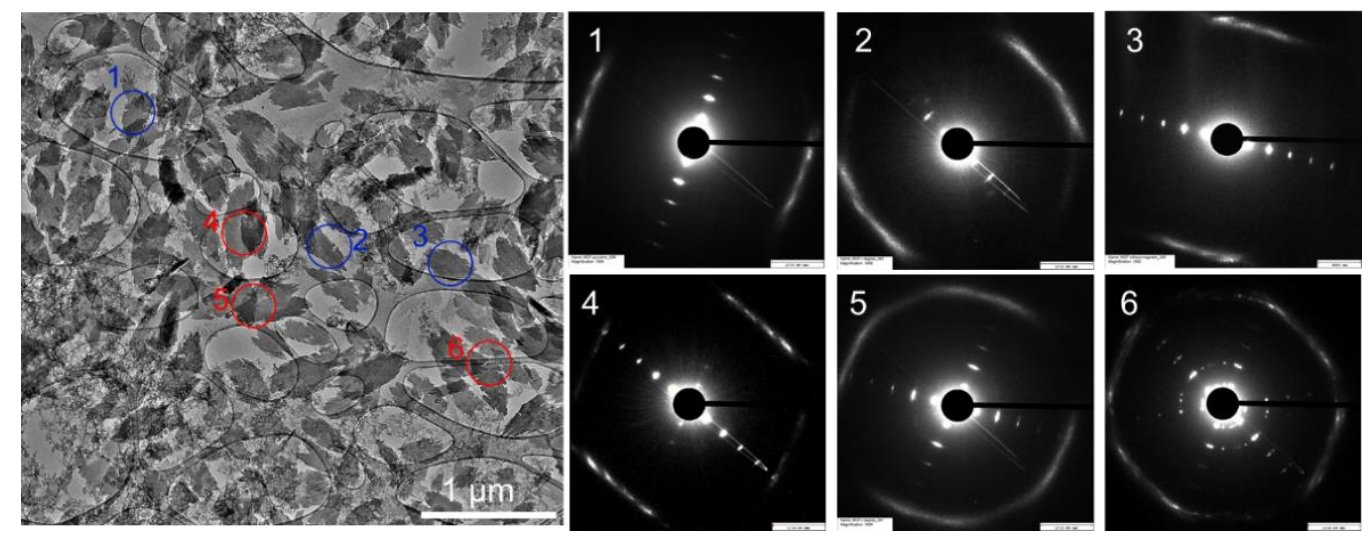

Figure S12. The SAED patterns obtained from isolated sheets. 


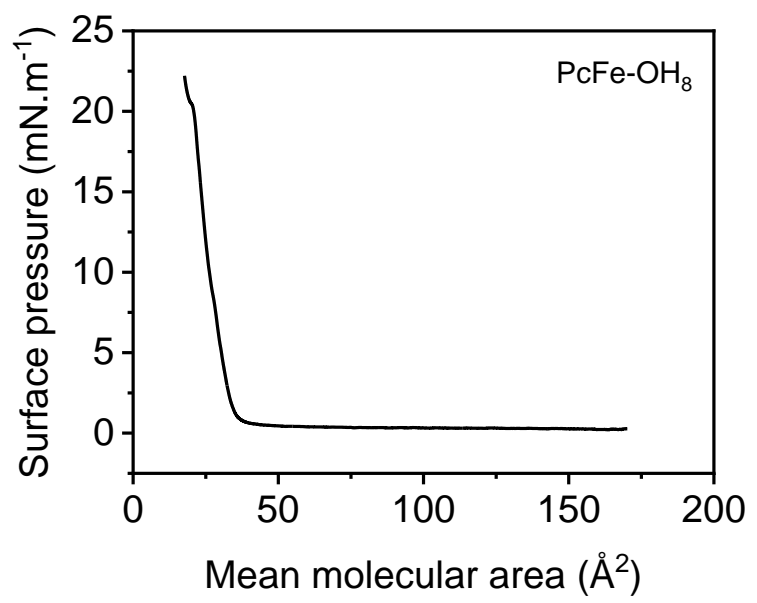

Figure S13. $\pi$-A isotherm for $\mathrm{PcFe}^{-\mathrm{OH}_{8} .} 50 \mu \mathrm{l}$ freshly prepared solution of $\mathrm{PcFe}-\mathrm{OH}_{8}(0.45 \mathrm{mM})$ in chloroform/DMF (2:1 in volume) was spread onto the water surface. After 10 min evaporation of the solvent, the Delrin barriers were driven forward to compress $\mathrm{PcFe}-\mathrm{OH}_{8}$ on the water surface at a rate of $5 \mathrm{~mm} \mathrm{~min}^{-1}$ until the surface pressure reached $22 \mathrm{mN} \mathrm{m}^{-1}$. The small molecular area implies that the PcFe$\mathrm{OH}_{8}$ molecules stand vertically on the water surface. 
a

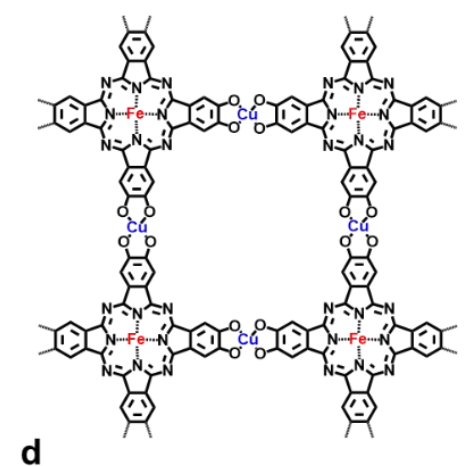

d

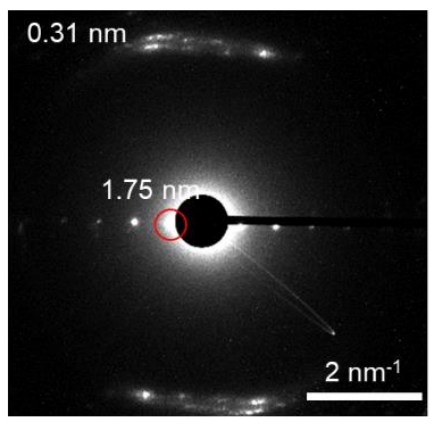

b
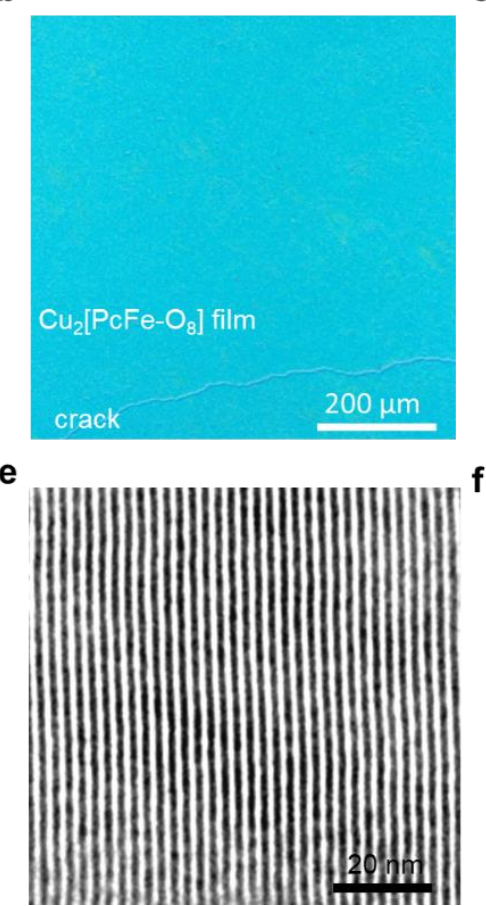

C
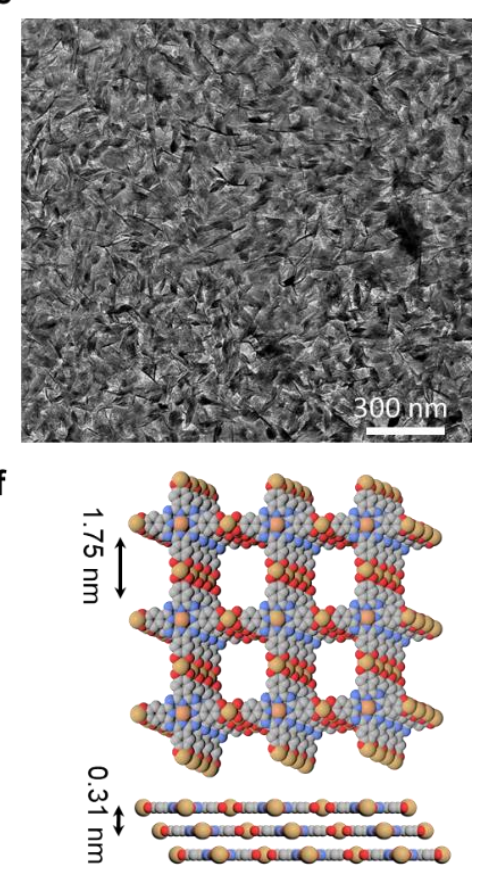

Figure S14. Crystal-structural characterization of the $\mathrm{Cu}_{2}\left[\mathrm{PcFe}-\mathrm{O}_{8}\right]$. (a) Scheme for $\mathrm{Cu}_{2}\left[\mathrm{PcFe}-\mathrm{O}_{8}\right]$. OM (b), and (c) TEM images of $\mathrm{Cu}_{2}\left[\mathrm{PcFe}-\mathrm{O}_{8}\right]$. (d) Corresponding SAED pattern. (e) HRTEM image of $\mathrm{Cu}_{2}\left[\mathrm{PcFe}-\mathrm{O}_{8}\right]$. (f) Crystal structure of $\mathrm{Cu}_{2}\left[\mathrm{PcFe}-\mathrm{O}_{8}\right]$. Grey, brown, red, blue, and white spheres represent $\mathrm{Cu}, \mathrm{Fe}, \mathrm{O}, \mathrm{N}$, and $\mathrm{C}$ atoms, respectively. 


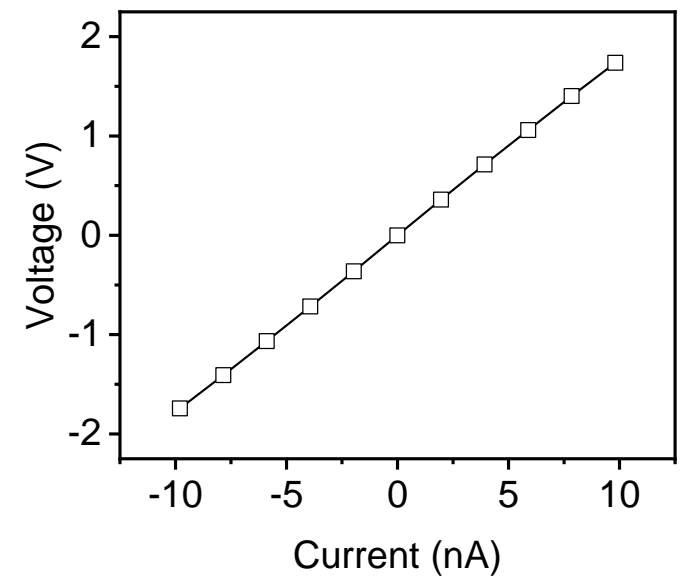

Figure S15. $I-V$ curve of $\mathrm{Cu}_{2}\left[\mathrm{PcCu}-\mathrm{O}_{8}\right]$ for van der Pauw measurement. 


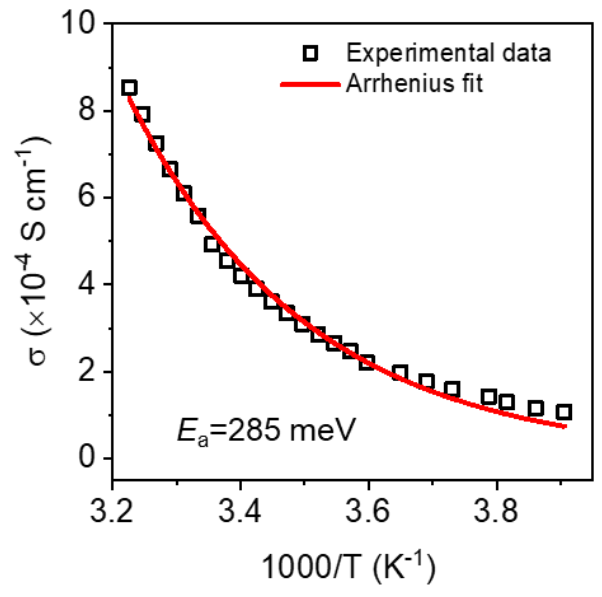

Figure S16. The plot of $\sigma$ versus the reciprocal of the temperature (1000/T). 


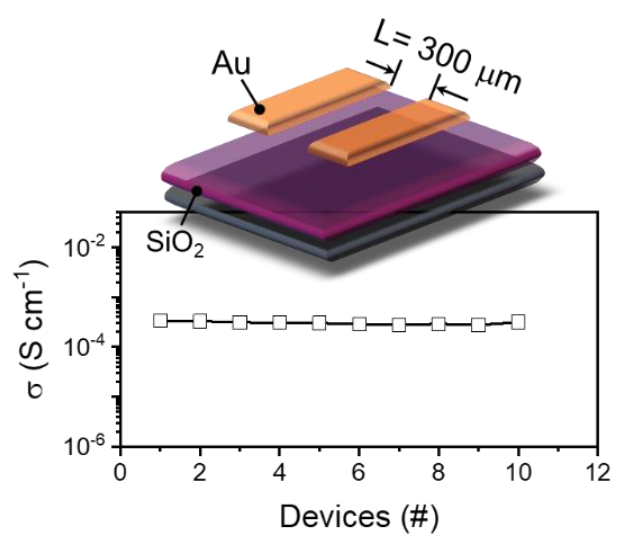

Figure S17. The conductivity of 10 two-probe devices with channel length of $300 \mu \mathrm{m}$. 


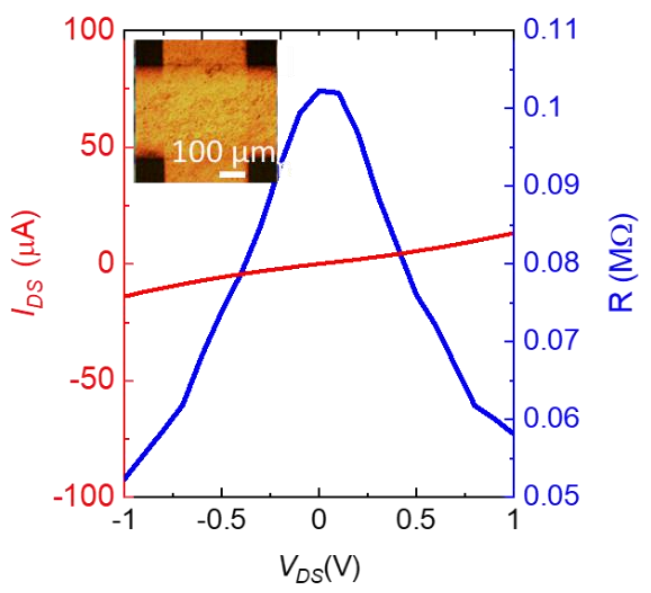

Figure S18. Measured $I-V$ curve for the vertical device with a thickness of $20 \mathrm{~nm}$. Inset: optical microscopy image of the vertical device; the device area is determined as $\sim 1.3 \times 10^{-3} \mathrm{~cm}^{2}$, i.e., the area overlapped between the top and bottom electrodes. Note that the resistance was measured by linear fitting of the $I-V$ curve in the voltage range from -1 to $+1 \mathrm{~V}$. 

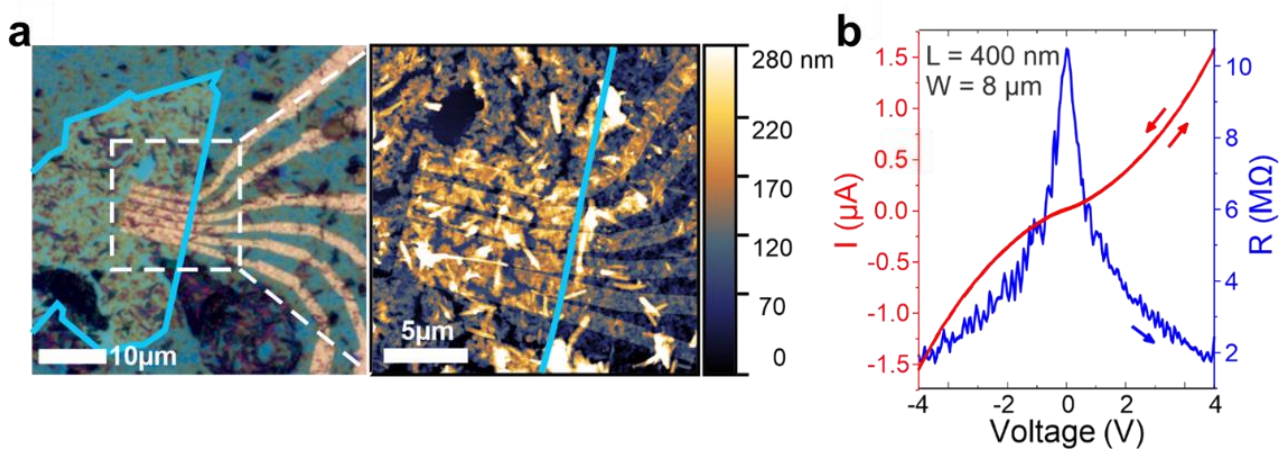

Figure S19. (a) Optical microscope image (left) and AFM image (right) of contact structures on one exemplary $h$-BN flake, which is marked in blue. Each flake contains several contacts. $I$ - $V$ curves were measured for each pair of neighboring contacts. (b) Measured $I-V$ curve and inverse of derivative (R) for lateral contacts with separation of $400 \mathrm{~nm}$. 

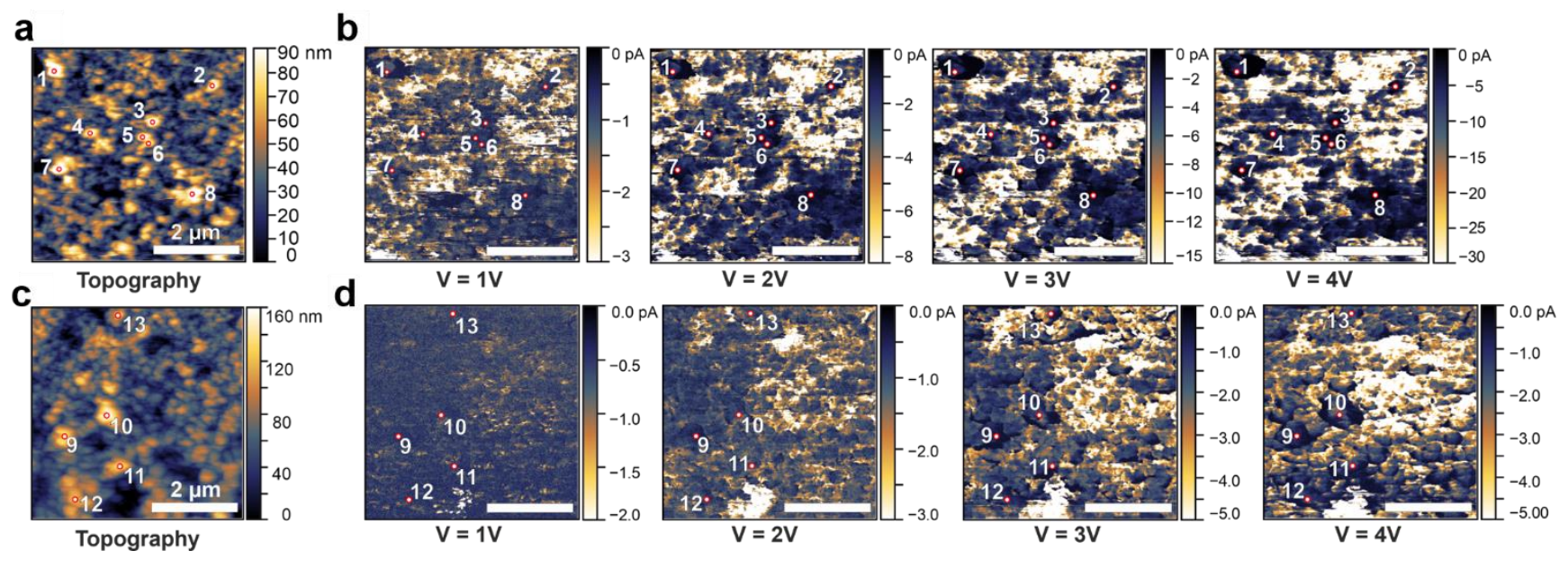

Figure S20. Topography of two arbitrarily chosen spots on the $\mathrm{Cu}_{2}\left[\mathrm{PcCu}-\mathrm{O}_{8}\right]$ film (a, c) and corresponding currents at the given applied voltages (b,d). The 8 and 5 highest points, respectively, from which the conductivity was derived as shown in Scheme 6, are labeled. 


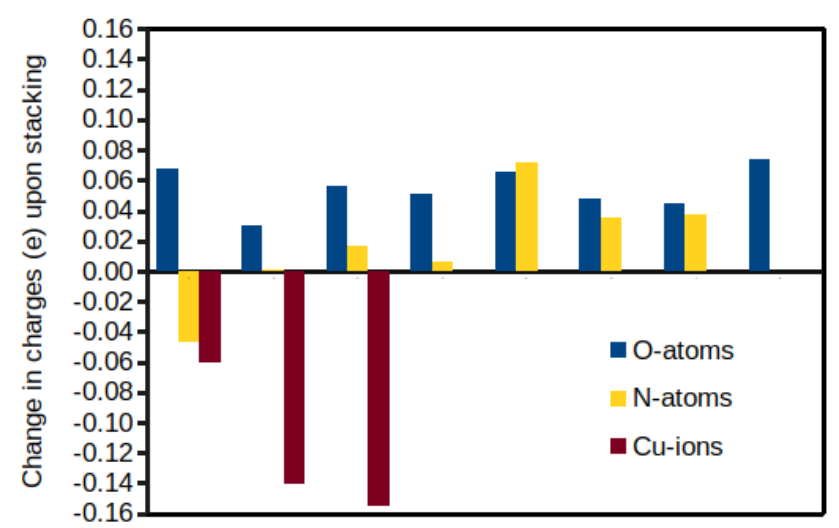

Figure S21. Changes in the calculated Bader atomic charges upon stacking. 
a

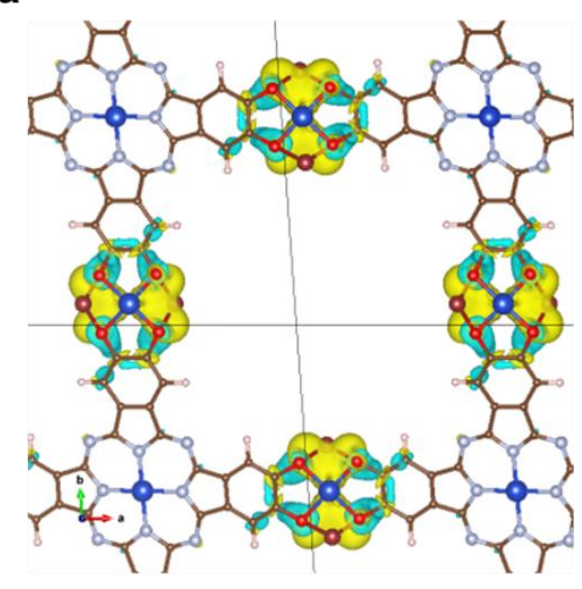

b

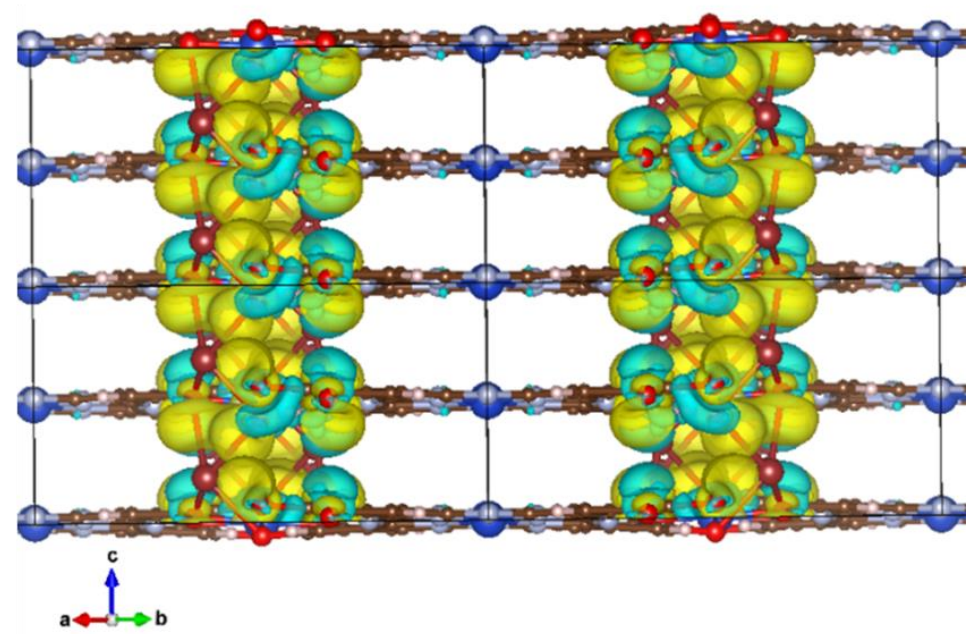

Figure S22. The charge density difference map of $\mathrm{Cu}_{2}\left[\mathrm{PcCu}-\mathrm{O}_{8}\right]$ multilayer with respect to the monolayer. (a) top view, (b) side view. Colour code: positive-yellow, negative-cyan. The charge density difference map shows, that the main charge density redistribution happens at the $\mathrm{Cu}-\mathrm{O}_{4}$ moiety, as it is demonsrated already by the Bader charge analisys. 


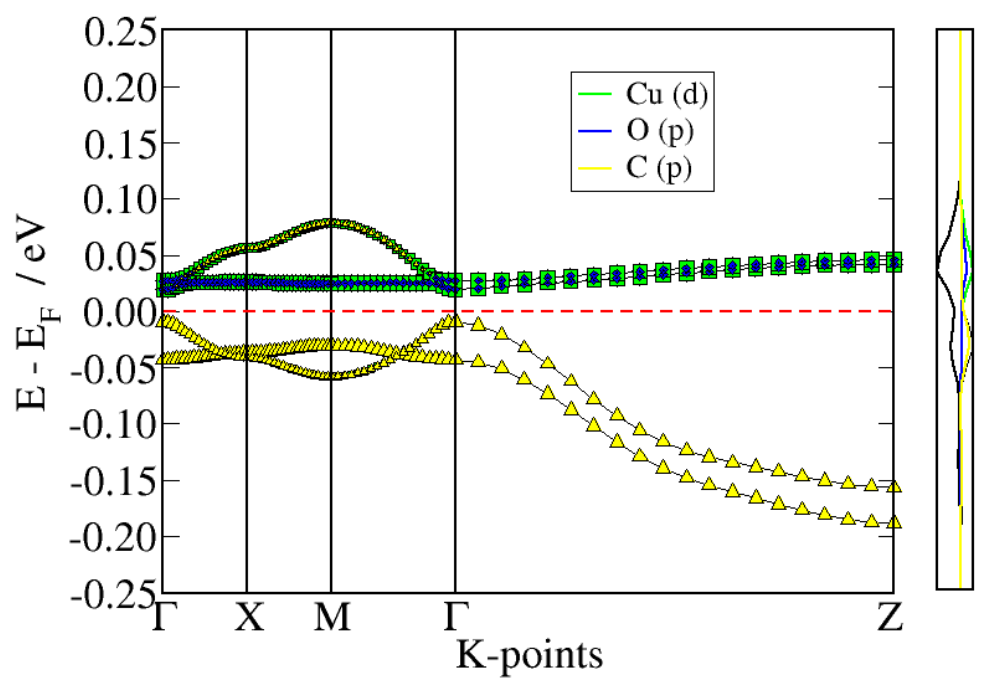

Figure S23. The fat bands show main contributions from $\mathrm{O}, \mathrm{C}$ and $\mathrm{Cu}$ at the band edges. $\mathrm{Cu}(\mathrm{d})$ levels and $\mathrm{O}(\mathrm{p})$ levels overlap and form the conductive bands, while the $\mathrm{C}(\mathrm{p})$ levels are below the Fermi level and form the edge of the valence bands. 
Table S1. Interfacial synthetic MOF thin films.

\begin{tabular}{|c|c|c|c|c|c|}
\hline MOFs & Linker/build units & Configuration & Method & Thickness & Ref \\
\hline CoTCPP-py-Cu & $-\mathrm{COOH} / \mathrm{Cu}^{2+}$ & face-on & $\mathrm{LB}^{[\mathrm{c}]}$ & monolayer & 20 \\
\hline NAFS-1 & $-\mathrm{COOH} / \mathrm{Cu}^{2+}$ & face-on & LB & monolayer & 21 \\
\hline $\mathrm{H}_{2} \mathrm{TCPP} \_\mathrm{Cu}$ & $-\mathrm{COOH} / \mathrm{Cu}^{2+}$ & face-on & LB & monolayer & 22 \\
\hline THTNi & $-\mathrm{SH} / \mathrm{Ni}^{2+}$ & face-on & LB & $0.7-0.9$ & 23 \\
\hline THTA-Co & $-\mathrm{SH}\left(\mathrm{NH}_{2}\right) / \mathrm{Co}^{2+}$ & face-on & LB & $0.8 \mathrm{~nm}$ & 24 \\
\hline$\left[\mathrm{Cu}_{2} \mathrm{I}_{2}(\mathrm{TAA})\right]_{\mathrm{n}}$ & $-\mathrm{I}, \mathrm{S} / \mathrm{Cu}^{2+}$ & face-on & petri dish & 4 & 25 \\
\hline N1 & Pyridine $/ \mathrm{Zn}^{2+}$ & face-on & vial & $1.2 \mathrm{~nm}$ & 26 \\
\hline FeNi-mono & $-\mathrm{CN} / \mathrm{Ni}^{2+}$ & face-on & LB & Monolayer & 27 \\
\hline nano-1 & $-\mathrm{SH} / \mathrm{Ni}^{2+}$ & face-on & beaker & $0.6 \mathrm{~nm}$ & 28 \\
\hline $\mathrm{Cu}-\mathrm{BHT}$ & $-\mathrm{SH} / \mathrm{Cu}^{2+}$ & face-on & $\mathrm{L} / \mathrm{L}^{[\mathrm{d}]}$ & $15-500 \mathrm{~nm}$ & 29 \\
\hline $\mathrm{Ag}-\mathrm{BHT}$ & $-\mathrm{SH} / \mathrm{Ag}^{+}$ & face-on & $\mathrm{L} / \mathrm{L}$ & $\sim 1 \mu \mathrm{m}$ & 30 \\
\hline PtBHT & $-\mathrm{SH} / \mathrm{Pt}^{2+}$ & face-on & $\mathrm{L} / \mathrm{L}$ & $0.6 \mathrm{~nm}$ & 31 \\
\hline $\mathrm{Co}_{3}(\mathrm{BHT})_{2}$ & $-\mathrm{SH} / \mathrm{Co}^{2+}$ & random & $\mathrm{L} / \mathrm{L}$ & $360 \mathrm{~nm}$ & 32 \\
\hline $\mathrm{Ag}_{3}(\mathrm{BHT})_{2}$ & $-\mathrm{SH} / \mathrm{Ag}^{+}$ & random & $\mathrm{L} / \mathrm{L}$ & $\sim 276 \mathrm{~nm}$ & 33 \\
\hline $\mathrm{Au}_{3}(\mathrm{BHT})_{2}$ & $-\mathrm{SH} / \mathrm{Au}^{+}$ & random & $\mathrm{L} / \mathrm{L}$ & $\sim 324 \mathrm{~nm}$ & 33 \\
\hline Ni-HAB & $-\mathrm{NH}_{2} / \mathrm{Ni}^{2+}$ & face-on & $\mathrm{L} / \mathrm{L}$ & $10 \mathrm{~nm}$ & 34 \\
\hline $\mathrm{Cu}-\mathrm{HAB}$ & $-\mathrm{NH}_{2} / \mathrm{Cu}^{2+}$ & face-on & $\mathrm{L} / \mathrm{L}$ & $11 \mathrm{~nm}$ & 34 \\
\hline Co-HAB & $-\mathrm{NH}_{2} / \mathrm{Co}^{2+}$ & face-on & $\mathrm{L} / \mathrm{L}$ & $13 \mathrm{~nm}$ & 34 \\
\hline $\mathrm{Cu}-\mathrm{CAT}-1$ & $-\mathrm{OH} / \mathrm{Cu}^{2+}$ & face-on & $\mathrm{L} / \mathrm{L}$ & $40 \mathrm{~nm}$ & 35 \\
\hline $\mathrm{Fe}_{3}(\mathrm{THT})_{2}\left(\mathrm{NH}_{4}\right)_{3}$ & $-\mathrm{SH} / \mathrm{Fe}^{2+}$ & face-on & $\mathrm{L} / \mathrm{L}$ & $\begin{array}{ll}20 & \mathrm{~nm}-2 \\
\mu \mathrm{m} & \end{array}$ & 36 \\
\hline $\mathrm{Ni}_{3}(\mathrm{HITP})_{2}$ & $-\mathrm{NH}_{2} / \mathrm{Ni}^{2+}$ & random & vial & $100 \mathrm{~nm}$ & 37 \\
\hline $\mathrm{Cu}_{3}(\mathrm{HHTP})_{2}$ & $-\mathrm{OH} / \mathrm{Cu}^{2+}$ & face-on & $\begin{array}{l}\text { on solid } \\
\text { surface }\end{array}$ & $50-250 \mathrm{~nm}$ & 38 \\
\hline $\mathrm{Cu}_{2}\left[\mathrm{PcCu}-\mathrm{O}_{8}\right]$ & $-\mathrm{OH} / \mathrm{Cu}^{2+}$ & edge-on & beaker & $20 \mathrm{~nm}$ & $\begin{array}{l}\text { This } \\
\text { work }\end{array}$ \\
\hline $\mathrm{Cu}_{2}\left[\mathrm{PcFe}-\mathrm{O}_{8}\right]$ & $-\mathrm{OH} / \mathrm{Cu}^{2+}$ & edge-on & beaker & $30 \mathrm{~nm}$ & $\begin{array}{l}\text { This } \\
\text { work }\end{array}$ \\
\hline
\end{tabular}

[a] $\mathrm{M}^{2+}=\mathrm{Co}^{2+}, \mathrm{Ru}^{2+}, \mathrm{Zn}^{2+}, \mathrm{Pb}^{2+}, \mathrm{Ni}^{2+}, \mathrm{Fe}^{2+}$. [b] Hexafunctional terpyridine. [c] Langmuir-Blodgett (LB) method. [d] Liquid/liquid (L/L) interface method. 
Table S2. Cell vectors and the optimized coordintaes for the multilayered $\left(\mathrm{Cu}_{2}\left[\mathrm{PcCu}-\mathrm{O}_{8}\right] 2 \mathrm{D} c-\mathrm{MOF}\right.$ in a POSCAR file format:

$\mathrm{C} \mathrm{Cu} \mathrm{H} \mathrm{N} \mathrm{NaO}$

1.00000000000000

\begin{tabular}{ccccccc}
18.1262239399999991 & 0.0000000000000000 & 0.0000000000000000 \\
-0.0012968900000000 & 18.1257430900000003 & 0.0000000000000000 \\
\multicolumn{3}{c}{0.9317485599999999} & \multicolumn{2}{c}{0.9318152300000000} & 3.3501589500000000 \\
$\mathrm{C}$ & $\mathrm{Cu}$ & $\mathrm{H}$ & $\mathrm{N}$ & $\mathrm{Na}$ & $\mathrm{O}$ & \\
32 & 3 & 8 & 8 & 4 & 8 &
\end{tabular}

Direct

$\begin{array}{llll}0.5989748529547967 & 0.3312881956294760 & 0.4373032762138749 \\ 0.5917393856770730 & 0.7816720580564152 & 0.5663689575724362 \\ 0.6002060585504339 & 0.2015163963110638 & 0.4038398729457953 \\ 0.5935824364207463 & 0.9126692436941255 & 0.5726912153496713 \\ 0.6200876050445245 & 0.4060295644688523 & 0.4724239624425479 \\ 0.6152506589956204 & 0.7061722947632774 & 0.5483155219827083 \\ 0.6393455662677781 & 0.2663476203247015 & 0.4193429651807605 \\ 0.6323619307827428 & 0.8465575951585649 & 0.5710287561882552 \\ 0.7067998340310311 & 0.4930202522347358 & 0.5416222122795986 \\ 0.7057610297482029 & 0.6154548638041106 & 0.5530491712644121 \\ 0.7810918941379654 & 0.5919303136603205 & 0.5757218589778645 \\ 0.7813423640231250 & 0.5142657550291574 & 0.5788382585349154 \\ 0.8459233055153561 & 0.4736924765420980 & 0.6045614364544889 \\ 0.8461964918430382 & 0.6328185968497877 & 0.5762091898421886 \\ 0.9122169661124033 & 0.5939201038876689 & 0.5812905061327100 \\ 0.9109902747340541 & 0.5131867776898815 & 0.6150838680572122 \\ 0.2016156597099652 & 0.6001646908097058 & 0.3984083239905587 \\ 0.2003061962873716 & 0.5194948716853034 & 0.4312254426829369 \\ 0.2669851133345205 & 0.6396154063871791 & 0.4056302779182204 \\ 0.2663761251341654 & 0.4805411089887812 & 0.4325919799010478 \\ 0.3317021324354386 & 0.5990152566259894 & 0.4268681463848409 \\ 0.3318530591459847 & 0.5213845195616571 & 0.4293342425827404 \\ 0.4065168137133739 & 0.6203756087294892 & 0.4600899370524587 \\ 0.4074526001742100 & 0.4979603768524186 & 0.4476732264263745\end{array}$




\begin{tabular}{|c|c|c|}
\hline 266037665618 & 0.2669044932092959 & 0.4279533014340231 \\
\hline 0.4740982849335538 & 0.8471479956248729 & 0.5803905285818303 \\
\hline 4982092652323544 & 0.4073820151436749 & 0.4499092194817749 \\
\hline 4933903191003779 & .7075116203905409 & 756530 \\
\hline & 718665 & 7199 \\
\hline & & \\
\hline 5217850427038186 & & 9592685 \\
\hline & & \\
\hline & & \\
\hline & & \\
\hline & & \\
\hline & & \\
\hline & & \\
\hline & & \\
\hline 0.8458684858634342 & 0.69363076 & 7667023 \\
\hline 1160977716482 & 78036069 & 5013 \\
\hline 211826578 & 0.41973921 & 6271 \\
\hline 6681 & 8649 & 1367 \\
\hline & 0.8476233690144781 & \\
\hline 0.5549074779615495 & 0.6628869083946967 & 0.52584589519 \\
\hline 0.5585618930862211 & 0.4506449613358328 & 0.472578947 \\
\hline 0.6624714748012366 & 0.5548474558859411 & 0.5332970927388985 \\
\hline 0.6882523003223966 & $\$ 30772527$ & 720079389 \\
\hline & 0.685584664 & \\
\hline 476 & 761 & 3309 \\
\hline & 496 & 5386 \\
\hline 74695658 & 0.5586405188075858 & 8965 \\
\hline 0.679573820 & 0.05 & 259 \\
\hline & & \\
\hline 0.0550560442624430 & 0.4340330450832539 & \\
\hline 0.4338925997281677 & 0.0565160475468360 & 0.1287113701760845 \\
\hline 0.6359496681250079 & 0.1357284619685615 & 0.3797692884065569 \\
\hline 0.6306955095749700 & 0.981 & 0.53 \\
\hline 0.9766581309567570 & 0.4773750884023542 & 22979514769054 \\
\hline & & $\begin{array}{l}0.5397671949315992 \\
39\end{array}$ \\
\hline
\end{tabular}



$0.1357563730494107 \quad 0.6359454138569944 \quad 0.3755998698716354$
$\begin{array}{lll}0.1314709966749419 & 0.4822485161043915 & 0.4755765199678450\end{array}$
$\begin{array}{llll}0.4827470452676081 & 0.1322833095870664 & 0.4646243584668781\end{array}$
$\begin{array}{llll}0.4774582495570172 & 0.9776754212633350 & 0.6216972677000356\end{array}$ 


\section{References}

(1) Kresse, G.; Furthmüller, J., Efficiency of ab-initio total energy calculations for metals and semiconductors using a plane-wave basis set. Comput. Mater. Sci. 1996, 6 (1), 15-50.

(2) Choudhuri, I.; Truhlar, D. G., HLE17: An efficient way to predict band gaps of complex materials. The Journal of Physical Chemistry C 2019, 123 (28), 17416-17424.

(3) Kresse, G.; Joubert, D., From ultrasoft pseudopotentials to the projector augmented-wave method. Phys. Rev. B 1999, 59 (3), 1758-1775.

(4) Perdew, J. P.; Chevary, J. A.; Vosko, S. H.; Jackson, K. A.; Pederson, M. R.; Singh, D. J.; Fiolhais, C., Atoms, molecules, solids, and surfaces: applications of the generalized gradient approximation for exchange and correlation. Phys. Rev. B 1992, 46 (11), 6671-6687.

(5) Perdew, J. P.; Burke, K.; Ernzerhof, M., Generalized gradient approximation made simple. Phys. Rev. Lett. 1996, 77 (18), 3865-3868.

(6) Anisimov, V. I.; Aryasetiawan, F.; Lichtenstein, A. I., First-principles calculations of the electronic structure and spectra of strongly correlated systems: theLDA+Umethod. J. Phys.: Condens. Matter 1997, 9 (4), 767-808.

(7) Li, W.; Sun, L.; Qi, J.; Jarillo-Herrero, P.; Dincă, M.; Li, J., High temperature ferromagnetism in $\pi$ conjugated two-dimensional metal-organic frameworks. Chem. Sci. 2017, 8 (4), 2859-2867.

(8) Monkhorst, H. J.; Pack, J. D., Special points for brillouin-zone integrations. Phys. Rev. B 1976, 13 (12), 5188-5192.

(9) Grimme, S., Semiempirical GGA-type density functional constructed with a long-range dispersion correction. J. Comput. Chem. 2006, 27 (15), 1787-1799.

(10) Yu, M.; Trinkle, D. R., Accurate and efficient algorithm for Bader charge integration. The Journal of Chemical Physics 2011, 134 (6), 064111.

(11) Chwang, R.; Smith, B. J.; Crowell, C. R., Contact size effects on the van der Pauw method for resistivity and Hall coefficient measurement. Solid State Electron. 1974, 17 (12), 1217-1227.

(12) Rietveld, G.; Koijmans, C. V.; Henderson, L. C.; Hall, M. J.; Harmon, S.; Warnecke, P.; Schumacher, B., DC conductivity measurements in the Van der Pauw geometry. IEEE. Trans. Instrum. Meas. 2003, 52 (2), 449-453.

(13) Van der Pauw, L., Philips Res. Rep., 13 \{1958) 1. Philips Tech. Rev 1958, 20, 320.

(14) Winkler, M. T.; Recht, D.; Sher, M. J.; Said, A. J.; Mazur, E.; Aziz, M. J., Insulator-to-metal transition in sulfur-doped silicon. Phys. Rev. Lett. 2011, 106 (17), 178701.

(15) Jiang, L.; Weber, J.; Puglisi, F. M.; Pavan, P.; Larcher, L.; Frammelsberger, W.; Benstetter, G.; Lanza, M., Understanding current instabilities in conductive atomic force microscopy. Materials 2019, 12 (3), 459.

(16) Nečas, D.; Klapetek, P., Gwyddion: an open-source software for SPM data analysis. Open Phys. 2012, 10 (1), 181-188.

(17) Ding, X.; Feng, X.; Saeki, A.; Seki, S.; Nagai, A.; Jiang, D., Conducting metallophthalocyanine 2D covalent organic frameworks: the role of central metals in controlling $\pi$-electronic functions. Chem. Commun. 2012, 48 (71), 8952-8954.

(18) Mineo, P.; Alicata, R.; Micali, N.; Villari, V.; Scamporrino, E., Water-soluble star polymers with a phthalocyanine as the core and poly (ethylene glycol) chains as branches. J. Appl. Polym. Sci. 2012, 126 (4), 1359-1368.

(19) Nagatomi, H.; Yanai, N.; Yamada, T.; Shiraishi, K.; Kimizuka, N., Synthesis and electric properties of a two-dimensional metal-organic framework based on phthalocyanine. Chem. Eur. J. 2018, 24 (8), 1806-1810.

(20) Makiura, R.; Motoyama, S.; Umemura, Y.; Yamanaka, H.; Sakata, O.; Kitagawa, H., Surface nanoarchitecture of a metal-organic framework. Nat. Mater. 2010, 9 (7), 565-571.

(21) Rubio-Giménez, V. c.; Tatay, S.; Volatron, F.; Martínez-Casado, F. J.; Martí-Gastaldo, C.; Coronado, E., High-quality metal-organic framework ultrathin films for electronically active interfaces. J. Am. Chem. Soc. 2016, 138 (8), 2576-2584.

(22) Motoyama, S.; Makiura, R.; Sakata, O.; Kitagawa, H., Highly crystalline nanofilm by layering of 
porphyrin metal-organic framework sheets. J. Am. Chem. Soc. 2011, 133 (15), 5640-5643.

(23) Dong, R.; Pfeffermann, M.; Liang, H.; Zheng, Z.; Zhu, X.; Zhang, J.; Feng, X., Large-area, freestanding, two-dimensional supramolecular polymer single-layer sheets for highly efficient electrocatalytic hydrogen evolution. Angew. Chem., Int. Ed. 2015, 54 (41), 12058-12063.

(24) Dong, R.; Zheng, Z.; Tranca, D. C.; Zhang, J.; Chandrasekhar, N.; Liu, S.; Zhuang, X.; Seifert, G.; Feng, X., Immobilizing molecular metal dithiolene-diamine complexes on 2D metal-organic frameworks for electrocatalytic $\mathrm{H}_{2}$ production. Chem. Eur. J. 2017, 23 (10), 2255-2260.

(25) Moreno-Moreno, M.; Troyano, J.; Ares, P.; Castillo, O.; Nijhuis, C. A.; Yuan, L.; Amo-Ochoa, P.; Delgado, S.; Gómez-Herrero, J.; Zamora, F., One-pot preparation of mechanically robust, transparent, highly conductive, and memristive metal-organic ultrathin film. ACS Nano 2018, 12 (10), 10171-10177. (26) Sakamoto, R.; Hoshiko, K.; Liu, Q.; Yagi, T.; Nagayama, T.; Kusaka, S.; Tsuchiya, M.; Kitagawa, Y.; Wong, W.-Y.; Nishihara, H., A photofunctional bottom-up bis (dipyrrinato) zinc (II) complex nanosheet. Nat. Commun. 2015, 6, 6713.

(27) Culp, J. T.; Park, J.-H.; Meisel, M. W.; Talham, D. R., Monolayer, bilayer, multilayers: evolving magnetic behavior in langmuir-blodgett films containing a two-dimensional iron-nickel cyanide square grid network. Inorg. Chem. 2003, 42 (9), 2842-2848.

(28) Kambe, T.; Sakamoto, R.; Kusamoto, T.; Pal, T.; Fukui, N.; Hoshiko, K.; Shimojima, T.; Wang, Z.; Hirahara, T.; Ishizaka, K.; Hasegawa, S.; Liu, F.; Nishihara, H., Redox control and high conductivity of nickel bis (dithiolene) complex $\pi$-nanosheet: a potential organic two-dimensional topological insulator. $J$. Am. Chem. Soc. 2014, 136 (41), 14357-14360.

(29) Huang, X.; Sheng, P.; Tu, Z.; Zhang, F.; Wang, J.; Geng, H.; Zou, Y.; Di, C.; Yi, Y.; Sun, Y.; Xu, W.; Zhu, D., A two-dimensional $\pi$-d conjugated coordination polymer with extremely high electrical conductivity and ambipolar transport behaviour. Nat. Commun. 2015, 6, 7408.

(30) Huang, X.; Li, H.; Tu, Z.; Liu, L.; Wu, X.; Chen, J.; Liang, Y.; Zou, Y.; Yi, Y.; Sun, J.; Xu, W.; Zhu, D., Highly conducting neutral coordination polymer with infinite two-dimensional silver-sulfur networks. J. Am. Chem. Soc. 2018, 140 (45), 15153-15156.

(31) Pal, T.; Doi, S.; Maeda, H.; Wada, K.; Tan, C. M.; Fukui, N.; Sakamoto, R.; Tsuneyuki, S.; Sasaki, S.; Nishihara, H., Interfacial transmetallation synthesis of a platinadithiolene nanosheet as a potential 2D topological insulator. Chem. Sci. 2019, 10 (20), 5218-5225.

(32) Clough, A. J.; Yoo, J. W.; Mecklenburg, M. H.; Marinescu, S. C., Two-dimensional metal-organic surfaces for efficient hydrogen evolution from water. J. Am. Chem. Soc. 2015, 137 (1), 118-121.

(33) Chen, I.-F.; Lu, C.-F.; Su, W.-F., Highly conductive 2D metal-organic framework thin film fabricated by liquid-liquid interfacial reaction using one-pot-synthesized benzenehexathiol. Langmuir 2018, 34 (51), 15754-15762.

(34) Lahiri, N.; Lotfizadeh, N.; Tsuchikawa, R.; Deshpande, V. V.; Louie, J., Hexaaminobenzene as a building block for a family of 2D coordination polymers. J. Am. Chem. Soc. 2017, 139 (1), 19-22.

(35) Rubio-Giménez, V.; Galbiati, M.; Castells-Gil, J.; Almora-Barrios, N.; Navarro-Sánchez, J.; Escorcia-Ariza, G.; Mattera, M.; Arnold, T.; Rawle, J.; Tatay, S.; Coronado, E.; Martí-Gastaldo, C., Bottom-up fabrication of semiconductive metal-organic framework ultrathin films. Adv. Mater. 2018, 30 (10), 1704291.

(36) Dong, R.; Han, P.; Arora, H.; Ballabio, M.; Karakus, M.; Zhang, Z.; Shekhar, C.; Adler, P.; Petkov, P. S.; Erbe, A.; Mannsfeld, S. C. B.; Felser, C.; Heine, T.; Bonn, M.; Feng, X.; Cánovas, E., High-mobility band-like charge transport in a semiconducting two-dimensional metal-organic framework. Nat. Mater. 2018, 17 (11), 1027-1032.

(37) Wu, G.; Huang, J.; Zang, Y.; He, J.; Xu, G., Porous field-effect transistors based on a semiconductive metal-organic framework. J. Am. Chem. Soc. 2016, 139 (4), 1360-1363.

(38) Song, X.; Wang, X.; Li, Y.; Zheng, C.; Zhang, B.; Di, C.-a.; Li, F.; Jin, C.; Mi, W.; Chen, L.; Hu, W., 2D semiconducting metal-organic framework thin films for organic spin valves. Angew. Chem., Int. Ed. 2020, 59 (3), 1118-1123. 